ESAIM: M2AN 46 (2012) 389-410

DOI: $10.1051 / \mathrm{m} 2 \mathrm{an} / 2011043$
ESAIM: Mathematical Modelling and Numerical Analysis

www.esaim-m2an.org

\title{
A COMPACTNESS RESULT FOR A SECOND-ORDER VARIATIONAL DISCRETE MODEL
}

\author{
Andrea Braides ${ }^{1}$, Anneliese Defranceschi ${ }^{2}$ And Enrico Vitali ${ }^{3}$
}

\begin{abstract}
We analyze a nonlinear discrete scheme depending on second-order finite differences. This is the two-dimensional analog of a scheme which in one dimension approximates a free-discontinuity energy proposed by Blake and Zisserman as a higher-order correction of the Mumford and Shah functional. In two dimension we give a compactness result showing that the continuous problem approximating this difference scheme is still defined on special functions with bounded hessian, and we give an upper and a lower bound in terms of the Blake and Zisserman energy. We prove a sharp bound by exhibiting the discrete-to-continuous $\Gamma$-limit for a special class of functions, showing the appearance new 'shear' terms in the energy, which are a genuinely two-dimensional effect.
\end{abstract}

Mathematics Subject Classification. 49J45, 49Q20, 68U10, 65D19, 65M06.

Received January 17, 2011. Revised June 22, 2011

Published online November 23, 2011.

\section{INTRODUCTION}

Since the seminal works by Geman and Geman [31], Blake and Zisserman [8] and Mumford and Shah [33], an extensive and fruitful research has been carried out in the field of variational models in image processing, and more in general in the study of the so-called free-discontinuity functionals. These functionals are characterized by an interplay between a surface energy localized on an unknown lower-dimensional set $K$ and a bulk term depending on an unknown function $u$, which is sufficiently smooth outside $K$.

The first-order (i.e., depending on the first derivatives of $u$ ) prototypical free-discontinuity energy is the celebrated Mumford and Shah functional:

$$
\operatorname{MS}(u, K)=\int_{\Omega \backslash K}|\nabla u|^{2} \mathrm{~d} x+\alpha \mathcal{H}^{1}(K \cap \Omega)
$$

(here $\Omega$ is a two-dimensional set and $\mathcal{H}^{1}$ is the one-dimensional Hausdorff measure). In the field of computer vision, given a function $g$, interpreted as the 'grey-level function' of an input image on a bidimensional region

Keywords and phrases. Computer vision, finite-difference schemes, gamma-convergence, free-discontinuity problems.

1 Dipartimento di Matematica, Università di Roma 'Tor Vergata', via della Ricerca Scientifica, 00133 Rome, Italy. braides@mat . uniroma2 . it

2 Dipartimento di Matematica, Università di Trento, via Sommarive 14, 38123 Povo, Italy.

3 Dipartimento di Matematica 'F. Casorati', Università di Pavia, via Ferrata 1, 27100 Pavia, Italy. 
$\Omega$, one seeks to find an output image as a minimizer of

$$
\min \left\{\operatorname{MS}(u, K)+\mu \int_{\Omega \backslash K}|u-g|^{2} \mathrm{~d} x\right\}
$$

over all closed sets $K$ of $\bar{\Omega}$ and all $u \in C^{1}(\Omega \backslash K)$. Such a pair $(u, K)$ gives an "optimal" balance between: (i) detecting the discontinuities $K$ of the image due to the edges of the objects (since the second term in MS penalizes the length $\mathcal{H}^{1}(K)$ of $K$ ); (ii) cancelling the discontinuities due to noise and small irregularities (by the smoothing effect of the gradient term), and (iii) being close to the datum $g$. In this respect, the (positive) coefficients $\mu$ and $\alpha$ are related to a characteristic length for smoothing and to a contrast threshold.

Minimization of free-discontinuity problems can be obtained by an 'indirect' approach, first obtaining weak solutions by applying the so-called direct methods of the Calculus of Variations to analogous energies defined on SBV spaces, where $K$ is interpreted as the discontinuity set of the function $u$. To that end, a rather complete theory of lower-semicontinuous first-order energies defined on SBV spaces has been developed, in which more elaborated functionals are included. At the same time, a number of approximations of the Mumford-Shah functional have been proposed (approximations by elliptic functionals on Sobolev spaces, finite element and finite difference schemes, higher-order perturbations, non-local methods, etc.) in order to overcome the issues arising from the difficulty of treating lower-dimensional interfacial energies and to make the numerical computation of minimizers possible (see e.g. Braides [12]).

A simple energy related to finite-difference schemes which approximates the Mumford-Shah functional has been derived from the model of Blake and Zissermann by Chambolle [25]. In a one-dimensional setting, it consists in considering

$$
E_{\varepsilon}(u)=\sum_{i} \varepsilon \psi_{\varepsilon}\left(\frac{u_{i}-u_{i-1}}{\varepsilon}\right),
$$

where $\left(u_{i}\right)$ is a discretization of $u$ on a grid of mesh-size $\varepsilon$, while

$$
\psi_{\varepsilon}(t)=\min \left(t^{2}, \frac{\gamma}{\varepsilon}\right)
$$

(with $\gamma>0$ a given parameter) is a truncated quadratic potential. In this formulation, if the difference of the grey-level values $u_{i}$ and $u_{i-1}$ of two adjacent pixels is beyond the threshold $\sqrt{\varepsilon \gamma}$, then the related energy gives the fixed penalization $\gamma$, whereas we have the (discretization of the) squared gradient otherwise. The scaling of the energy density is exactly conceived in a way that the contribution of this second type of interactions has the dimension of a surface energy (in one dimension this translates into just counting the number of discontinuities). The discrete functionals $E_{\varepsilon}$ are proved to be asymptotically equivalent to the continuous one, the parameter $\gamma$ playing the same role as $\alpha$.

In two dimensions (and higher) a similar result holds when nearest-neighbor interactions on a square grid are considered (see Chambolle [26]). In that case, the surface energy must be adapted to take into account the anisotropy of the discrete model, substituting $\mathcal{H}^{1}(K \cap \Omega)$ by a surface integral

$$
\int_{K \cap \Omega}\|\nu\|_{1} \mathrm{~d} \mathcal{H}^{1}
$$

where $\nu$ is the normal to $K$ and $\|\nu\|_{1}=\left|\nu_{1}\right|+\left|\nu_{2}\right|$. Note that the energies $E_{\varepsilon}$ are still 'of the same order' of the Mumford-Shah energy since their limit is sandwiched between MS and $\sqrt{2}$ MS. In order to overcome the anisotropy due to the lattice symmetries, various corrections have been proposed taking into account long-range interactions (Braides and Gelli [15], Chambolle [27]), adapted grids for finite-elements (Chambolle and Dal Maso [28]), averaged quantities (Bourdin and Chambolle [9]), or random interactions (Braides and Piatnitski [16]). From a standpoint different from that of image processing, the same type of asymptotic analysis is of fundamental interest in physics and continuum mechanics when energies of the same form as $E_{\varepsilon}$ are 
considered, with $\psi_{\varepsilon}$ some type of interatomic potential (e.g., Lennard-Jones potentials), and the goal is to derive a corresponding continuous theory. In many cases the truncated quadratic case has proved not to be a mere prototype but a fundamental building block for general energies (e.g., in the case of Lennard-Jones interatomic potential - see Braides et al. [17]). In that context, complex and possibly anisotropic energy densities in the continuous approximation are of great interest, highlighting critical phenomena at an atomistic level. Such energies arise also for truncated quadratic potential as above when many-points interactions are taken into account in the vector case (see Alicandro et al. [2]), which are necessary e.g. when we want to approximate general fracture energies in the context of linear elasticity (for the derivation of linear elasticity with no fracture energy from geometrically nonlinear quadratic lattice interactions see Braides et al. [18] and Schmidt [35]).

In this paper we will present the asymptotic analysis of a discrete scheme with many-point interactions related to the second-order free-discontinuity energy analogous to MS also introduced by Blake and Zisserman [8]; namely, the functional

$$
\mathrm{BZ}_{\alpha, \beta}\left(u, K_{0}, K_{1}\right)=\int_{\Omega \backslash\left(K_{0} \cup K_{1}\right)}\left|\nabla^{2} u\right|^{2} \mathrm{~d} x \mathrm{~d} y+\alpha \mathcal{H}^{1}\left(K_{0} \cap \Omega\right)+\beta \mathcal{H}^{1}\left(\left(K_{1} \backslash K_{0}\right) \cap \Omega\right)
$$

where $u$ belongs to $C^{0}\left(\Omega \backslash K_{0}\right) \cap C^{2}\left(\Omega \backslash\left(K_{0} \cup K_{1}\right)\right)$, and the sets $K_{0}$ and $K_{1} \backslash K_{0}$ represent the set of discontinuity points for $u$ and $\nabla u$, respectively; the constants $\alpha, \beta>0$ satisfy $\beta \leq \alpha \leq 2 \beta$ for semicontinuity reasons. A different choice for the leading second-order term $\left|\nabla^{2} u\right|^{2}$ is the square laplacian $|\Delta u|^{2}$; indeed, any linear combination of these two is a suitable (rotationally invariant) second-order energy density (see $[8,10,32])$. These functionals were introduced to overcome some drawbacks of first-order models such as the over-segmentation of steep gradients (ramp effect), or the inadequacy in detecting "crease discontinuities". For the existence of minimizers of $\mathrm{BZ}_{\alpha, \beta}$ and an in-depth analysis of their properties we mainly refer to a series of papers by Carriero et al. (see, for instance, the recent works [22-24] and the cited references therein).

In the one-dimensional case the discretization of the functional (1.2) consists in an energy taking into account the discretization of the second derivative, exactly of the same form as (1.1):

$$
E_{\varepsilon}(u)=\sum_{i} \varepsilon \psi_{\varepsilon}\left(\frac{u_{i+1}-2 u_{i}+u_{i-1}}{\varepsilon^{2}}\right) .
$$

For these energies we have three different regimes: when the argument of the truncated quadratic potential is of order 1 (corresponding in the limit to the second derivative of $u$ ), of order $1 / \varepsilon$ (allowing for 'creases'; i.e., discontinuities of the first derivative), and of order $1 / \varepsilon^{2}$ (allowing for 'jumps', i.e., discontinuities of the function $u$ itself). The $\Gamma$-limit (see Braides [14]) is a one-dimensional version of (1.2); i.e.,

$$
F\left(u, K_{0}, K_{1}\right)=\int_{\Omega \backslash\left(K_{0} \cup K_{1}\right)}\left|\nabla^{2} u\right|^{2} \mathrm{~d} x+2 \gamma \#\left(K_{0} \cap \Omega\right)+\gamma \#\left(\left(K_{1} \backslash K_{0}\right) \cap \Omega\right)
$$

(in this case $F=\mathrm{BZ}_{2 \gamma, \gamma} ;$ i.e., $\alpha=2 \gamma$ and $\beta=\gamma$. To obtain arbitrary $\alpha$ and $\beta$, slightly more complex energy density can be considered). Our work is related to the extension of the one-dimensional discrete-to-continuous result above to the two-(and higher-)dimensional case, by considering energies

$$
E_{\varepsilon}(u)=\sum_{i, j} \varepsilon^{2} \psi_{\varepsilon}\left(\sqrt{V_{i, j}^{\varepsilon}}\right)
$$

where $\psi_{\varepsilon}$ is as above, the sum ranges over all the nodes of a square grid of mesh-size $\varepsilon$, while $V_{i, j}^{\varepsilon}$ is the value, on the node $(i, j)$, of a suitable discrete form of

$$
u_{x x}^{2}+2 u_{x y}^{2}+u_{y y}^{2}
$$

(coinciding with $\left(u_{i+1}-2 u_{i}+u_{i-1} / \varepsilon^{2}\right)^{2}$ in the one-dimensional case). 
The main results of the paper are a compactness result for functionals such as $E_{\varepsilon}$ (see Sect. 5) and a comparison with the continuous case by giving upper and lower estimates on the surface energy of the $\Gamma$-limit in terms of $F:=\mathrm{BZ}_{2 \gamma, \gamma}$. Namely, we show that (suitably interpolated) sequences $\left(u_{\varepsilon}\right)$ bounded in $L^{2}$ and with equibounded energies $E_{\varepsilon}\left(u_{\varepsilon}\right)$ admit a converging subsequence for which the (weak form of the) functional $F\left(u, K_{0}, K_{1}\right)$ is finite. The proof differs from the one-dimensional one, since it is not possible to directly interpolate the functions $u_{\varepsilon}$ in such a way that $E_{\varepsilon}\left(u_{\varepsilon}\right)=F\left(u_{\varepsilon}, K_{0}^{\varepsilon}, K_{1}^{\varepsilon}\right)\left(K_{0}^{\varepsilon}, K_{1}^{\varepsilon}\right.$ the 'jump' and 'crease' sets of $u_{\varepsilon}$, respectively), which was the key argument in [14]. It is however possible to construct an interpolation such that $E_{\varepsilon}\left(u_{\varepsilon}\right) \geq F\left(u_{\varepsilon}, K_{0}^{\varepsilon}, K_{1}^{\varepsilon}\right)$, which provides at the same time the compactness result and a lower bound for the energy. We then give an upper bound of the $\Gamma$-limit with $c F\left(u, K_{0}, K_{1}\right)$, for $c$ a (large) constant. We give an estimate for this constant $c$ and we compute exactly the $\Gamma$-limit in some special situations. In particular, for example if $S_{u}$ and $S_{\nabla u}$ are composed of segments parallel to the coordinate axes, then there exists the $\Gamma$-limit

$$
\Gamma-\lim _{\varepsilon \rightarrow 0} E_{\varepsilon}(u)=\int_{\Omega}\left|\nabla^{2} u\right|^{2} \mathrm{~d} x \mathrm{~d} y+2 \gamma \mathcal{H}^{1}\left(S_{u}\right)+\gamma \mathcal{H}^{1}\left(S_{\langle\nabla u, \nu\rangle} \backslash S_{u}\right)+2 \gamma \mathcal{H}^{1}\left(S_{\left\langle\nabla u, \nu^{\perp}\right\rangle}\right),
$$

where $S_{\langle\nabla u, \nu\rangle}$ is the subset of $S_{\nabla u}$ where the orthogonal component $\langle\nabla u, \nu\rangle$ of $\nabla u$ is discontinuous, and $S_{\left\langle\nabla u, \nu^{\perp}\right\rangle}$ is the subset of $S_{\nabla u}$ (possibly not disjoint from the previous one) where the tangential component $\left\langle\nabla u, \nu^{\perp}\right\rangle$ of $\nabla u$ is discontinuous. Note that the last term describes a 'shear' contribution, which is a genuinely higherdimensional effect.

Even though the computation of the limit is partial, our result shows the 'asymptoticity' of the discrete energies to the Blake-Zisserman functional, and provides a new approximation which adds up to its only other approximation, with elliptic energies, proposed by Ambrosio et al. [6] (see also [7]) following the celebrated Ambrosio-Tortorelli approximation of the Mumford-Shah functional (see [3,4]). It is likely that, for computational purposes, the anisotropies of the limit, even though not precisely described, can be corrected in the same way as has been done for the discrete approximation of the Mumford-Shah functional (and recalled above).

The exact determination of the $\Gamma$-limit of $\left(E_{\varepsilon}\right)$ appears to be a technically demanding task, due to the complex interaction with the underlying mesh. In fact, the discrete nature of the energies implies that discontinuity sets (especially, of the first gradient) will in general have a 'zig-zag' microscopic geometry (even when the limit continuous function has a crease along a straight segment, if this is not oriented along the coordinate directions), which generates large second gradients. A main technical point that we have not been able to overcome is to adapt to this situation the techniques that allow to modify boundary values without increasing the limit energy. This has been shown to be possible for first-order problems by Alicandro and Cicalese [1], but for second-order energies we face difficulties that resemble those that are encountered in the theory of solid-solid phase transitions (see Conti et al. [30]), complicated by the complex multi-scale interactions at the microscopic level. A further difficulty is related to the lack of a general lower-semicontinuity theory for second-order freediscontinuity functionals (see [11] for the one-dimensional theory, and [34] for some remarks in the general case), and the corresponding lack of comparison energies other than the Blake-Zisserman functional. Note that the interfacial energy in (1.4) is indeed of the form

$$
\int_{S_{u} \cup S_{\nabla u}} \varphi\left(u^{ \pm}, \nabla^{ \pm} u, \nu\right) \mathrm{d} \mathcal{H}^{1}
$$

for which no lower-semicontinuity results are present in the literature.

\section{Preliminaries}

We will use standard notation for the Sobolev spaces $H^{1}(\Omega)=W^{1,2}(\Omega)$ and $H^{2}(\Omega)=W^{2,2}(\Omega)$ on an open subset $\Omega$ of $\mathbb{R}^{n}$. We denote by $B V(\Omega)$ the space of real-valued functions of bounded variation, i.e. the space of functions $u \in L^{1}(\Omega)$ whose distributional derivative is representable by a measure in $\Omega$, i.e.

$$
\int_{\Omega} u \frac{\partial \varphi}{\partial x_{i}} \mathrm{~d} x=-\int_{\Omega} \varphi \mathrm{d} D_{i} u \quad \text { for every } \varphi \in C_{c}^{\infty}(\Omega) \text { and } i=1, \ldots, n
$$


for some $\mathbb{R}^{n}$-valued Borel measure $D u=\left(D_{1} u, \ldots, D_{n} u\right)$ on $\Omega$. Let $D u=D^{a} u+D^{s} u$ be the Lebesgue decomposition of $D u$ into absolutely continuous and singular part, and let $\nabla u$ be the density of $D^{a} u$. We denote by $S B V(\Omega)$ (special functions with bounded variation) the space of functions in $B V(\Omega)$ such that $D^{s} u$ is a $(n-1)$-rectifiable measure, i.e. there exist a rectifiable set $S_{u}$ and a Borel function

$$
\left(u^{+}, u^{-}, \nu\right): S_{u} \rightarrow \mathbb{R} \times \mathbb{R} \times S^{n-1}
$$

which gives the following representation:

$$
D^{s} u=\left(u^{+}-u^{-}\right) \nu_{u} \mathcal{H}^{n-1}\left\llcorner S_{u}\right.
$$

where $\mathcal{H}^{n-1}$ stands for the $(n-1)$-dimensional Hausdorff measure $\left(u^{ \pm}\right.$are the traces of $u$ on the approximate discontinuity set $S_{u}$; for the precise definitions we refer to [5]). We will also need the following space:

$$
G S B V(\Omega)=\left\{u: \Omega \rightarrow \mathbb{R}: u^{M}:=(-M) \vee u \wedge M \in S B V(\Omega) \text { for every } M>0\right\} .
$$

It turns out that for any $u \in G S B V(\Omega)$ an approximate gradient $\nabla u$ can be defined with the property that

$$
\nabla u=\nabla u^{M} \quad \text { a.e. where } u=u^{M} \text {, and } \nabla u=0 \text { a.e. where }|u|>M .
$$

We set $S_{\nabla u}=\bigcup_{i} S_{\nabla_{i} u}\left(\right.$ where $\nabla_{i} u$ is the $i$ th component of $\left.\nabla u\right)$.

Moreover, we define

$$
G S B V^{2}(\Omega)=\left\{u \in G S B V(\Omega): \quad \nabla u \in[G S B V(\Omega)]^{n}\right\} .
$$

If $u \in G S B V^{2}(\Omega)$ we denote by $\nabla^{2} u$ the matrix whose $i$ th row is the approximate gradient of the $G S B V$ function $\nabla_{i} u$; hence, $\left(\nabla^{2} u\right)_{i j}=\nabla_{j}\left(\nabla_{i} u\right)=: \nabla_{i j}^{2} u$. We will also refer to $\nabla^{2} u$ as the approximate hessian (matrix) of $u$.

When $n=1$ we write $u^{\prime}$ and $u^{\prime \prime}$ instead of $\nabla u$ and $\nabla^{2} u$, respectively, while for $n=2$ we will usually use $\nabla_{x} u, \nabla_{y} u, \nabla_{x x}^{2} u$ instead of $\nabla_{1} u, \nabla_{2} u, \nabla_{11}^{2}$, and so on.

Finally, we recall some basic properties of the one-dimensional sections of $B V$ functions; they will be applied in Section 6 to prove two-dimensional estimates from one-dimensional results (slicing). Let $\Omega \subseteq \mathbb{R}^{2}$ be an open set, and $u: \Omega \rightarrow \mathbb{R}$. For every $y \in \mathbb{R}$ let

$$
\Omega^{y}=\{x \in \mathbb{R}:(x, y) \in \Omega\}, \quad u^{y}: \Omega^{y} \rightarrow \mathbb{R}, \quad u^{y}(x)=u(x, y) .
$$

For the following results we refer, for instance, to $[5,19]$.

Theorem 2.1. Let $w \in G S B V_{l o c}(\Omega)$. Then for a.e. $y \in \mathbb{R}$, with $\Omega^{y} \neq \emptyset$,

(i) $w^{y} \in G S B V\left(\Omega^{y}\right)$;

(ii) $\left(w^{y}\right)^{\prime}(x)=\nabla_{x} w(x, y)$ for a.e. $x \in \Omega^{y}$;

(iii) $S_{w^{y}}=\left(S_{w}\right)^{y}$.

In particular, if $w \in G S B V^{2}(\Omega)$, then for a.e. $y \in \mathbb{R}$

$$
\left(w^{y}\right)^{\prime \prime}(x)=\nabla_{x x}^{2} w(x, y) \quad \text { for a.e. } x \in \Omega^{y} .
$$

\section{THE DISCRETE SECOND-ORDER ENERGY}

Before displaying the precise definition of the energy functionals $E_{\varepsilon}$, we introduce some notation.

If $\alpha=\left(\alpha_{1}, \alpha_{2}\right) \in(\mathbb{N} \cup\{0\})^{2}$ is a multiindex, we denote by $|\alpha|=\alpha_{1}+\alpha_{2}$ its length. For every $\varepsilon>0$, and for any given real-valued function $u$ on a subset of $\mathbb{R}^{2}$, we define the difference quotients $\Delta_{\varepsilon}^{\alpha} u$ when $|\alpha| \leq 2$ as follows. We set $\Delta_{\varepsilon}^{\alpha} u=u$ if $|\alpha|=0$, and

$$
\begin{aligned}
& \Delta_{\varepsilon}^{(1,0)} u(x, y)=\varepsilon^{-1}[u(x+\varepsilon, y)-u(x, y)], \\
& \Delta_{\varepsilon}^{(0,1)} u(x, y)=\varepsilon^{-1}[u(x, y+\varepsilon)-u(x, y)],
\end{aligned}
$$


for any $(x, y) \in \mathbb{R}^{2}$ where the right-hand sides are defined. Moreover, we set:

$$
\begin{aligned}
& \Delta_{\varepsilon}^{(2,0)} u(x, y)=\varepsilon^{-1}\left[\Delta_{\varepsilon}^{(1,0)} u(x, y)-\Delta_{\varepsilon}^{(1,0)} u(x-\varepsilon, y)\right] \\
&=\varepsilon^{-2}[u(x+\varepsilon, y)+u(x-\varepsilon, y)-2 u(x, y)], \\
& \Delta_{\varepsilon}^{(1,1)} u(x, y)=\varepsilon^{-1}\left[\Delta_{\varepsilon}^{(1,0)} u(x, y+\varepsilon)-\Delta_{\varepsilon}^{(1,0)} u(x, y)\right]=\varepsilon^{-2}[u(x+\varepsilon, y+\varepsilon)+u(x, y)-u(x+\varepsilon, y)-u(x, y+\varepsilon)] \\
&=\varepsilon^{-1}\left[\Delta_{\varepsilon}^{(0,1)} u(x+\varepsilon, y)-\Delta_{\varepsilon}^{(0,1)} u(x, y)\right], \\
& \Delta_{\varepsilon}^{(0,2)} u(x, y)=\varepsilon^{-1}\left[\Delta_{\varepsilon}^{(0,1)} u(x, y)-\Delta_{\varepsilon}^{(0,1)} u(x, y-\varepsilon)\right] \\
&=\varepsilon^{-2}[u(x, y+\varepsilon)+u(x, y-\varepsilon)-2 u(x, y)] .
\end{aligned}
$$

In the continuous setting we will use $D^{\alpha}$ as the usual differential operator determined by the multiindex $\alpha$.

If $A$ is a subset of $\mathbb{R}^{2}$ we set

$$
A_{\varepsilon}=\left\{(x, y) \in \varepsilon \mathbb{Z}^{2}:(x, y)+\varepsilon(\sigma, \tau) \in \text { Afor all } \sigma, \tau \in\{-1,0,1\}\right\} .
$$

We now introduce the second-order discrete energy functional $E_{\varepsilon}$ whose behaviour will be studied as $\varepsilon \rightarrow 0$. If $u$ is a real-valued function on a subset $A$ of $\mathbb{R}^{2}$ we define

$$
E_{\varepsilon}(u, A)=\sum_{z \in A_{\varepsilon}} \varepsilon^{2}\left(\psi_{\varepsilon}\left(\Delta_{\varepsilon}^{(2,0)} u(z)\right)+2 \psi_{\varepsilon}\left(\Delta_{\varepsilon}^{(1,1)} u(z)\right)+\psi_{\varepsilon}\left(\Delta_{\varepsilon}^{(0,2)} u(z)\right)\right)
$$

where

$$
\psi_{\varepsilon}(t)=\min \left(t^{2}, \gamma / \varepsilon\right)
$$

and $\gamma>0$ is a fixed parameter.

In Section 5 we prove that any sequence $\left(u_{\varepsilon_{k}}\right)$, on which $E_{\varepsilon_{k}}$ are equibounded, is precompact with respect to a suitable convergence. Clearly, in this respect, the same result can be applied e.g. to the functionals

$$
\sum_{z \in A_{\varepsilon}} \varepsilon^{2} \psi_{\varepsilon}\left(\sqrt{\left(\Delta_{\varepsilon}^{(2,0)} u\right)^{2}+2\left(\Delta_{\varepsilon}^{(1,1)} u\right)^{2}+\left(\Delta_{\varepsilon}^{(0,2)} u\right)^{2}}\right)
$$

(where the difference quotients are computed at $z$ ). Indeed, for every $a, b, c \geq 0$ it turns out that:

$$
\frac{1}{4}\left[\psi_{\varepsilon}(a)+2 \psi_{\varepsilon}(b)+\psi_{\varepsilon}(c)\right] \leq \psi_{\varepsilon}\left(\sqrt{a^{2}+2 b^{2}+c^{2}}\right) \leq \psi_{\varepsilon}(a)+2 \psi_{\varepsilon}(b)+\psi_{\varepsilon}(c) .
$$

In Section 6 we give estimates for $\Gamma$-upper and lower limits of the energies $E_{\varepsilon}$ (Thms. 6.1 and 6.2). From those results, in particular we may compare $\Gamma$-limits with the anisotropic Blake and Zisserman functionals defined as

$$
\mathrm{BZ}_{\alpha, \beta}^{1}(u, \Omega)=\int_{\Omega}\left|\nabla^{2} u\right|^{2} \mathrm{~d} x \mathrm{~d} y+\alpha \int_{S_{u} \cap \Omega}\|\nu\|_{1} \mathrm{~d} \mathcal{H}^{1}+\beta \int_{\left(S_{\nabla u} \backslash S_{u}\right) \cap \Omega}\|\nu\|_{1} \mathrm{~d} \mathcal{H}^{1}
$$

as follows.

Theorem 3.1. Let $E_{\varepsilon}$ be defined by (3.2) and let $\Omega$ be a bounded open set in $\mathbb{R}^{2}$. Then we have

$$
\Gamma-\liminf _{\varepsilon \rightarrow 0} E_{\varepsilon}(u, \Omega) \geq B Z_{2 \gamma, \gamma}^{1}(u, \Omega)
$$

moreover, if $\Omega$ is star-shaped we have

$$
\Gamma-\limsup _{\varepsilon \rightarrow 0} E_{\varepsilon}(u, \Omega) \leq B Z_{24 \sqrt{2} \gamma, 24 \sqrt{2} \gamma}^{1}(u, \Omega) .
$$


Note that neither of these estimates is sharp; in particular a better lower estimate is exhibited in Theorem 6.1 by taking into account also 'shear' contributions, which provide a lower-bound energy of a different form than $\mathrm{BZ}_{\alpha, \beta}^{1}$; this lower estimate is sharp for $u$ with discontinuity sets composed of segments in the coordinate directions (see Thm. 6.4).

The study of the one-dimensional analog of (3.2) was addressed by Braides in [14]. In particular he proved the following result, which will be needed in the slicing argument in Section 6 . Let $J$ be a bounded interval and $\mathcal{H}^{2}(J)$ be the space of piecewise- $H^{2}$ functions on $J$. On $\mathcal{H}^{2}(J)$ define

$$
\mathcal{E}(u)=\int_{J}\left|u^{\prime \prime}\right|^{2} \mathrm{~d} t+\gamma \#\left(S_{u^{\prime}} \backslash S_{u}\right)+2 \gamma \# S_{u} .
$$

Let now $\varepsilon>0$; for any real-valued function $u$ on $J \cap \varepsilon \mathbb{Z}$ define

$$
\mathcal{E}_{\varepsilon}(u)=\sum_{\substack{x \in J \cap \in \mathbb{Z} \\ x \pm \varepsilon \in J}} \varepsilon \psi_{\varepsilon}\left(\frac{u(x+\varepsilon)+u(x-\varepsilon)-2 u(x)}{\varepsilon^{2}}\right) .
$$

Extend any function $u: J \cap \varepsilon \mathbb{Z} \rightarrow \mathbb{R}$ to the whole $\varepsilon \mathbb{Z}$ with value 0 outside $J$, and then to all of $\mathbb{R}$ by setting $u(x)=u(\varepsilon\lfloor x / \varepsilon\rfloor)$ (here $\lfloor\cdot\rfloor$ denotes the integer part). We can thus consider the $L^{1}(J)$-convergence for sequences of functions $J \cap \varepsilon_{k} \mathbb{Z} \rightarrow \mathbb{R}$ as $\varepsilon_{k} \rightarrow 0$. From [14] we have the following result.

Theorem 3.2. Let $\left(\varepsilon_{k}\right)$ be a positive infinitesimal sequence. Then $\mathcal{E}_{\varepsilon_{k}} \Gamma$-converge to $\mathcal{E}$ (extended with value $+\infty$ on $\left.L^{1}(J) \backslash \mathcal{H}^{2}(J)\right)$, with respect to the $L^{1}(J)$ convergence on bounded sets of $L^{2}(J)$.

\section{EXTENSION OF FUNCTIONS}

For any given real-valued function $u$ on $\varepsilon \mathbb{Z}^{2}$ we now define an extension to $\mathbb{R}^{2}$ in such a way that the $L^{2}$-norm of the second gradient is controlled by the second difference quotients on the nodes in $\varepsilon \mathbb{Z}^{2}$.

Let $I$ be the set of multiindices $\{(0,0),(1,0),(0,1),(1,1)\}$. From the theory of finite elements we draw the so-called Bogner-Fox-Schmit rectangle:

Proposition 4.1 ([29], Thm. 2.2.14). Let $J_{1}$ and $J_{2}$ be bounded intervals. There exists a unique polynomial $p(x, y)$ of the form

$$
p(x, y)=\sum_{h, k=0}^{3} a_{h k} x^{h} y^{k}
$$

for which the values of $D^{\alpha} p$ at the vertices of the rectangle $J_{1} \times J_{2}$ are equal to prescribed values for every $\alpha \in I$.

Assume that at each point $z \in \varepsilon \mathbb{Z}^{2}$ we are given four values:

$$
\bar{p}^{\alpha}(z), \quad \text { for every } \alpha \in I .
$$

For each square $Q$ of the mesh (with side of length $\varepsilon$ ), by the above proposition there exists a unique polynomial $p_{Q}$ determined by the conditions

$$
D^{\alpha} p_{Q}(z)=\bar{p}^{\alpha}(z) \quad(\alpha \in I)
$$

when $z$ varies among the vertices of $Q$.

Proposition 4.2 ([29], Thm 2.2.15). If two squares $Q_{1}$ and $Q_{2}$ of $\varepsilon \mathbb{Z}^{2}$ share a side, then $p_{Q_{1}}=p_{Q_{2}}$ on this side.

Let $v: \mathbb{R}^{2} \rightarrow \mathbb{R}$ be the function defined by

$$
\left.v\right|_{Q}=p_{Q}
$$

for every $Q$ of the mesh. Then $v \in C^{1}\left(\mathbb{R}^{2}\right) \cap H_{\text {loc }}^{2}\left(\mathbb{R}^{2}\right)$. 
We now consider the case when the values $\bar{p}^{\alpha}(z)$ are difference quotients of a given function $u: \varepsilon \mathbb{Z}^{2} \rightarrow \mathbb{R}$. Therefore, we assume that

$$
\bar{p}^{\alpha}(z)=\left(\Delta_{\varepsilon}^{\alpha} u\right)(z), \quad \text { for every } z \in \varepsilon \mathbb{Z}^{2} \text { and } \alpha \in I .
$$

Definition 4.3. On account of Proposition 4.2 , for every $u: \varepsilon \mathbb{Z}^{2} \rightarrow \mathbb{R}$ we can define the function $S_{\varepsilon} u: \mathbb{R}^{2} \rightarrow \mathbb{R}$ as the unique function which coincides on each square $Q$ of the mesh determined by $\varepsilon \mathbb{Z}^{2}$ with the polynomial $p$ of the form (4.1) satisfying

$$
D^{\alpha} p(z)=\Delta_{\varepsilon}^{\alpha} u(z) \quad \text { for every } \alpha \in I
$$

on the vertices $z$ of $Q$.

We will estimate the $L^{2}$-norm of $D^{2} S_{\varepsilon} u$ for every square $Q$ of the mesh. Let $V_{0}$ be the set of the vertices of $Q_{0}:=[-1,1] \times[-1,1]$. Denote the centre of $Q$ by $z_{Q}=\left(x_{Q}, y_{Q}\right)$ and define

$$
u_{0}(z)=u\left(z_{Q}+\frac{\varepsilon}{2} z\right), \text { for } z \in V_{0} ; \quad p_{0}(z)=S_{\varepsilon} u\left(z_{Q}+\frac{\varepsilon}{2} z\right), \text { for } z \in Q_{0} .
$$

Then for every multiindex $\alpha$ and for every $v \in V_{0}$ we have (note that the length of the sides of $Q_{0}$ is 2 ):

$$
\Delta_{2}^{\alpha} u_{0}(v)=\left(\frac{\varepsilon}{2}\right)^{|\alpha|} \Delta_{\varepsilon}^{\alpha} u\left(z_{Q}+\frac{\varepsilon}{2} v\right) .
$$

Moreover, by the definition of $S_{\varepsilon} u$, for every $\alpha \in I$ and $v \in V_{0}$ :

$$
\left(\frac{\varepsilon}{2}\right)^{|\alpha|} \Delta_{\varepsilon}^{\alpha} u\left(z_{Q}+\frac{\varepsilon}{2} v\right)=\left(\frac{\varepsilon}{2}\right)^{|\alpha|} D^{\alpha} S_{\varepsilon} u\left(z_{Q}+\frac{\varepsilon}{2} v\right)=D^{\alpha} p_{0}(v) .
$$

Therefore, by the uniqueness of the polynomial in Proposition 4.1, $p_{0}$ can be determined as the polynomial of the form (4.1) satisfying the conditions

$$
D^{\alpha} p_{0}(v)=\Delta_{2}^{\alpha} u_{0}(v) \quad \text { for every } v \in V_{0} \text { and } \alpha \in I .
$$

Remark 4.4. The set of conditions (4.4) allows to express the coefficients $a_{h k}$ of $p_{0}$, for every square, in a linear way through the values $\Delta_{2}^{\alpha} u_{0}(v)$. In particular, this implies a pointwise estimate for the interpolating polynomial $S_{\varepsilon} u$. For every square $Q$ of the mesh $\varepsilon \mathbb{Z}^{2}$ let

$$
M(u, Q)=\max \left\{|u(x, y)|:(x, y) \in \varepsilon \mathbb{Z}^{2}, x-\bar{x}, y-\bar{y} \in\{-\varepsilon, 0, \varepsilon\} \text { for some vertex }(\bar{x}, \bar{y}) \text { of } Q\right\} .
$$

Then there exists $C>0$, independent of $u$, such that for every square $Q$

$$
\left|S_{\varepsilon} u\right| \leq C M(u, Q) \quad \text { on } Q
$$

Indeed, if $Q$ is such a square, then for every $(x, y) \in Q$

$$
S_{\varepsilon} u(x, y)=\sum_{h, k=0}^{3} a_{h k} \frac{\left(x-x_{Q}\right)^{h}\left(y-y_{Q}\right)^{k}}{(\varepsilon / 2)^{h+k}},
$$

where the coefficients $a_{h k}$ are bounded in terms of the values of $u_{0}$ on $Q_{0}$ and on the neighbouring squares, hence in terms of $M(u, Q)$. Moreover, $\left|x-x_{Q}\right|,\left|y-y_{Q}\right| \leq \varepsilon / 2$ on $Q$.

As mentioned above we have to estimate the $L^{2}$-norm of $D^{2} S_{\varepsilon} u$. Since

$$
\int_{Q}\left|D^{2} S_{\varepsilon} u\left(z^{\prime}\right)\right|^{2} \mathrm{~d} z^{\prime}=\frac{4}{\varepsilon^{2}} \int_{Q_{0}}\left|D^{2} p_{0}(z)\right|^{2} \mathrm{~d} z
$$

we focus on the corresponding estimate for $D^{2} p_{0}$. 
Proposition 4.5. Let $p_{0}$ be the unique polynomial of the form (4.1) satisfying condition (4.4), where $u_{0}$ is a given function for which the right-hand side is defined. Then the coefficients $a_{i j}$, for $i+j \geq 2$, depend in a linear way on the values of $u_{0}$ only through the difference quotients

$$
\Delta_{2}^{\alpha} u_{0}(v), \quad \text { for } v \in V_{0} \text { and }|\alpha|=2 .
$$

In particular, there exists a positive constant $C$, independent of $u_{0}$, such that

$$
\int_{Q_{0}}\left|D^{2} p_{0}(z)\right|^{2} \mathrm{~d} z \leq C \sum_{v \in V_{0}} \sum_{|\alpha|=2}\left|\Delta_{2}^{\alpha} u_{0}(v)\right|^{2} .
$$

Proof. Let $A=(1,1), B=(-1,1), C=(-1,-1), D=(1,-1)$ be the vertices of $Q_{0}$. As mentioned above, the set of conditions (4.4) allows to express the coefficients $a_{h k}$ of $p_{0}$ in a linear way through the difference quotients $\Delta_{2}^{\alpha} u_{0}(v)$. Since the symmetries of $Q_{0}$ reflect on the symmetries of these linear conditions, by addition/subtraction of suitable pairs of equations we easily get the following linear equations:

- the condition for $\alpha=(0,0)$ yields:

$$
\begin{aligned}
& \text { (1) } a_{33}+\left(a_{31}+a_{22}+a_{13}\right)+\left(a_{20}+a_{11}+a_{02}\right)+a_{00}=\frac{1}{2}\left(u_{0}(A)+u_{0}(C)\right), \\
& \text { (2) }\left(a_{32}+a_{23}\right)+\left(a_{30}+a_{21}+a_{12}+a_{03}\right)+\left(a_{10}+a_{01}\right)=\frac{1}{2}\left(u_{0}(A)-u_{0}(C)\right), \\
& \text { (3) }-a_{33}+\left(-a_{31}+a_{22}-a_{13}\right)+\left(a_{20}-a_{11}+a_{02}\right)+a_{00}=\frac{1}{2}\left(u_{0}(B)+u_{0}(D)\right), \\
& \text { (4) }\left(-a_{32}+a_{23}\right)+\left(-a_{30}+a_{21}-a_{12}+a_{03}\right)+\left(-a_{10}+a_{01}\right)=\frac{1}{2}\left(u_{0}(B)-u_{0}(D)\right) .
\end{aligned}
$$

- the condition for $\alpha=(1,0)$ yields (for ease of notation we denote $\Delta_{\varepsilon}^{(1,0)} u_{0}$ and $\Delta_{\varepsilon}^{(0,1)} u_{0}$, with $\varepsilon=2$, simply by $\delta^{1} u_{0}$ and $\delta^{2} u_{0}$, respectively):

$$
\begin{aligned}
& \text { (5) }\left(3 a_{32}+2 a_{23}\right)+\left(3 a_{30}+2 a_{21}+a_{12}\right)+a_{10}=\frac{1}{2}\left[\left(\delta^{1} u_{0}\right)(A)+\left(\delta^{1} u_{0}\right)(C)\right], \\
& \text { (6) } 3 a_{33}+\left(3 a_{31}+2 a_{22}+a_{13}\right)+\left(2 a_{20}+a_{11}\right)=\frac{1}{2}\left[\left(\delta^{1} u_{0}\right)(A)-\left(\delta^{1} u_{0}\right)(C)\right], \\
& \text { (7) }\left(3 a_{32}-2 a_{23}\right)+\left(3 a_{30}-2 a_{21}+a_{12}\right)+a_{10}=\frac{1}{2}\left[\left(\delta^{1} u_{0}\right)(B)+\left(\delta^{1} u_{0}\right)(D)\right], \\
& \text { (8) } 3 a_{33}+\left(3 a_{31}-2 a_{22}+a_{13}\right)+\left(-2 a_{20}+a_{11}\right)=\frac{1}{2}\left[\left(\delta^{1} u_{0}\right)(B)-\left(\delta^{1} u_{0}\right)(D)\right] .
\end{aligned}
$$

An analogous set corresponds to $\alpha=(0,1)$ (with $\delta^{2}$ in place of $\delta^{1}$ ).

- the conditions for $\alpha=(1,1)$ clearly have the right-hand sides which depend only on $\Delta_{2}^{(1,1)} u_{0}$ and do not involve $a_{h k}$ if $h+k \leq 1$. 
By replacing equations (1) and (3) with $\frac{1}{2}[(1) \pm(3)]$ and equations (2) and (4) with $\frac{1}{2}[(2) \pm(4)]$ we get

$$
\begin{array}{ll}
\left(1^{\prime}\right) & a_{22}+\left(a_{20}+a_{02}\right)+a_{00}=\beta_{1}, \\
\left(2^{\prime}\right) & a_{23}+\left(a_{21}+a_{03}\right)+a_{01}=\beta_{2}, \\
\left(3^{\prime}\right) & a_{33}+\left(a_{31}+a_{13}\right)+a_{11}=\beta_{3}, \\
\left(4^{\prime}\right) & a_{32}+\left(a_{30}+a_{12}\right)+a_{10}=\beta_{4},
\end{array}
$$

where

$$
\begin{aligned}
& \beta_{1}=\frac{1}{4}\left[u_{0}(A)+u_{0}(B)+u_{0}(C)+u_{0}(D)\right], \\
& \beta_{2}=\frac{1}{4}\left[u_{0}(A)+u_{0}(B)-u_{0}(C)-u_{0}(D)\right], \\
& \beta_{3}=\frac{1}{4}\left[u_{0}(A)-u_{0}(B)+u_{0}(C)-u_{0}(D)\right],=\left(\Delta_{2}^{(1,1)} u_{0}\right)(C), \\
& \beta_{4}=\frac{1}{4}\left[u_{0}(A)-u_{0}(B)-u_{0}(C)+u_{0}(D)\right] .
\end{aligned}
$$

The same argument on the set (5)-(8) yields:

$$
\begin{aligned}
& \left(5^{\prime}\right) \quad 3 a_{32}+\left(3 a_{30}+a_{12}\right)+a_{10}=\gamma_{1}, \\
& \left(6^{\prime}\right) \quad 3 a_{33}+\left(3 a_{31}+a_{13}\right)+a_{11}=\gamma_{2}, \\
& \left(7^{\prime}\right) \quad 2 a_{23}+2 a_{21}=\gamma_{3}, \\
& \left(8^{\prime}\right) \quad+2 a_{22}+2 a_{20}=\gamma_{4},
\end{aligned}
$$

where

$$
\begin{aligned}
& \gamma_{1}=\frac{1}{4}\left[\left(\delta^{1} u_{0}\right)(A)+\left(\delta^{1} u_{0}\right)(B)+\left(\delta^{1} u_{0}\right)(C)+\left(\delta^{1} u_{0}\right)(D)\right], \\
& \gamma_{2}=\frac{1}{4}\left[\left(\delta^{1} u_{0}\right)(A)+\left(\delta^{1} u_{0}\right)(B)-\left(\delta^{1} u_{0}\right)(C)-\left(\delta^{1} u_{0}\right)(D)\right]=\frac{1}{2}\left[\left(\Delta_{2}^{(1,1)} u_{0}\right)(D)+\left(\Delta_{2}^{(1,1)} u_{0}\right)(C)\right], \\
& \gamma_{3}=\frac{1}{4}\left[\left(\delta^{1} u_{0}\right)(A)-\left(\delta^{1} u_{0}\right)(B)+\left(\delta^{1} u_{0}\right)(C)-\left(\delta^{1} u_{0}\right)(D)\right]=\frac{1}{2}\left[\left(\Delta_{2}^{(2,0)} u_{0}\right)(A)-\left(\Delta_{2}^{(2,0)} u_{0}\right)(D)\right], \\
& \gamma_{4}=\frac{1}{4}\left[\left(\delta^{1} u_{0}\right)(A)-\left(\delta^{1} u_{0}\right)(B)-\left(\delta^{1} u_{0}\right)(C)+\left(\delta^{1} u_{0}\right)(D)\right]=\frac{1}{2}\left[\left(\Delta_{2}^{(2,0)} u_{0}\right)(A)+\left(\Delta_{2}^{(2,0)} u_{0}\right)(D)\right] .
\end{aligned}
$$

Through equations $\left(1^{\prime}\right),\left(2^{\prime}\right)$ and $\left(4^{\prime}\right)$ we can express $a_{00}, a_{01}$ and $a_{10}$ in terms of $a_{h k}$ for $h+k \geq 2$. Thus, we can eliminate $a_{10}$ from equation $\left(5^{\prime}\right)$ by means of equation $\left(4^{\prime}\right)$, and get

$$
\left(5^{\prime \prime}\right) \quad 2 a_{32}+2 a_{30}=\gamma_{1}-\beta_{4}=\frac{1}{2}\left[\left(\Delta_{2}^{(2,0)} u_{0}\right)(A)+\left(\Delta_{2}^{(2,0)} u_{0}\right)(D)\right]
$$

(the same can be done for the corresponding equation containing $a_{01}$ in the set of conditions for the derivative with respect to the second variable). Therefore, the resulting equations involving the terms $a_{h k}$ with $h+k \geq 2$ (i.e. Eqs. $\left(5^{\prime \prime}\right),\left(6^{\prime}\right),\left(7^{\prime}\right),\left(8^{\prime}\right)$, the analogous ones for the other derivative, the equations for the mixed derivative and $\left.\left(3^{\prime}\right)\right)$ have a right-hand side which can be expressed only through $\left(\Delta_{2}^{\alpha} u_{0}\right)$ for $|\alpha|=2$.

Remark 4.6. From the proof of Proposition 4.5 (see, in particular, Eqs. $\left(2^{\prime}\right)$ and $\left(4^{\prime}\right)$ ) we also get that $a_{h k}$ with $h+k=1$ can be expressed in terms of $\Delta_{2}^{\alpha} u_{0}(v)$ for $v \in V_{0}$ and $|\alpha| \geq 1$ (indeed $\beta_{2}$ and $\beta_{4}$ are sums of first-order difference quotients). 
Corollary 4.7. There exists a constant $C$ such that for every $u: \varepsilon \mathbb{Z}^{2} \rightarrow \mathbb{R}$ and for every square $Q$ of the mesh $\varepsilon \mathbb{Z}^{2}$ we have

$$
\int_{Q}\left|D^{2} S_{\varepsilon} u(z)\right|^{2} \mathrm{~d} z \leq C \sum_{z} \sum_{|\alpha|=2} \varepsilon^{2}\left|\Delta_{\varepsilon}^{\alpha} u(z)\right|^{2},
$$

where the first sum is performed on all $z$ vertices of $Q$.

Proof. By Proposition 4.5 and by (4.5) and (4.3), it turns out that:

$$
\begin{aligned}
\int_{Q}\left|D^{2} S_{\varepsilon} u(z)\right|^{2} \mathrm{~d} z & =\frac{4}{\varepsilon^{2}} \int_{Q_{0}}\left|D^{2} p_{0}(z)\right|^{2} \mathrm{~d} z \leq C \frac{4}{\varepsilon^{2}} \sum_{v \in V_{0}} \sum_{|\alpha|=2}\left|\left(\Delta_{2}^{\alpha} u_{0}\right)(v)\right|^{2} \\
& =\frac{1}{4} C \sum_{z \text { vertex of } Q} \sum_{|\alpha|=2} \varepsilon^{2}\left|\Delta_{\varepsilon}^{\alpha} u(z)\right|^{2} .
\end{aligned}
$$

\section{Compactness}

Let $\Omega$ be a bounded open subset of $\mathbb{R}^{2}$ with Lipschitz boundary. Let $u$ be a real-valued function on $\Omega \cap \varepsilon \mathbb{Z}^{2}$; by previously defining $u$ with value 0 on $\varepsilon \mathbb{Z}^{2} \backslash \Omega$, we can apply the procedure introduced in Section 4 and extend $u$ to a $C^{1}$ piecewise-polynomial function $S_{\varepsilon} u$ on $\mathbb{R}^{2}$ in such a way that the $L^{2}$-norm of $D^{2} S_{\varepsilon} u$ is controlled by the second difference quotients on the nodes in $\varepsilon \mathbb{Z}^{2}$ (see Cor. 4.7). The function $u$ also admits the trivial "pixel-like" piecewise-constant extension $T_{\varepsilon} u: \mathbb{R}^{2} \rightarrow \mathbb{R}$ defined by:

$$
T_{\varepsilon} u(x, y)=\hat{u}(x, y):=u\left(x^{\varepsilon}, y^{\varepsilon}\right), \quad \text { where } \quad\left(x^{\varepsilon}, y^{\varepsilon}\right):=(\varepsilon\lfloor x / \varepsilon\rfloor, \varepsilon\lfloor y / \varepsilon\rfloor)
$$

$(\lfloor\cdot\rfloor$ denotes the integer part).

In this section we prove a compactness result for the smooth and the piecewise-constant interpolations of sequences $\left(u_{\varepsilon_{k}}\right)$ for which the energies $E_{\varepsilon_{k}}\left(u_{\varepsilon_{k}}, \Omega\right)$ are equibounded, as well as their $L^{2}$-norms (this assumption is satisfied $e . g$. if a fidelity term to an $L^{2}$ input is added as for the Mumford-Shah functional).

Remark 5.1. Let us note that the $L^{2}$-norm of the smooth interpolation $S_{\varepsilon} u$ can be easily estimated by the $L^{2}$-norm of the piecewise-constant interpolation $T_{\varepsilon} u$ :

$$
\left\|S_{\varepsilon} u\right\|_{L^{2}\left(\mathbb{R}^{2}\right)} \leq C\left\|T_{\varepsilon} u\right\|_{L^{2}\left(\mathbb{R}^{2}\right)}
$$

for a suitable $C>0$ independent of $u$ (as above $u$ has value 0 on $\varepsilon \mathbb{Z}^{2} \backslash \Omega$ ). Indeed, in the notation of Remark 4.4 there exists a constant $C$ such that

$$
\left\|S_{\varepsilon} u\right\|_{L^{2}\left(\mathbb{R}^{2}\right)}^{2} \leq C \sum_{Q} \varepsilon^{2} M(u, Q)^{2},
$$

where the sum is taken over all squares $Q$ of the mesh $\varepsilon \mathbb{Z}^{2}$. By the definition of $M(u, Q)$ we get

$$
M(u, Q) \leq \sum\left\{|u(x, y)|:(x, y) \in \varepsilon \mathbb{Z}^{2}, x-\bar{x}, y-\bar{y} \in\{-\varepsilon, 0, \varepsilon\} \text { for some vertex }(\bar{x}, \bar{y}) \text { of } Q\right\} .
$$

Hence

$$
\sum_{Q} \varepsilon^{2} M(u, Q)^{2} \leq 16 \sum_{(x, y) \in \varepsilon \mathbb{Z}^{2}} \varepsilon^{2} u(x, y)^{2}=16\left\|T_{\varepsilon} u\right\|_{L^{2}\left(\mathbb{R}^{2}\right)}^{2}
$$

(as a consequence of the application of (5.2) for every $Q$, each node is considered 16 times). 
Theorem 5.2. Let $\left(\varepsilon_{k}\right)$ be a positive infinitesimal sequence and let $\left(u_{k}\right)$ be a sequence of functions $\Omega_{\varepsilon_{k}} \rightarrow \mathbb{R}$. Assume that

$$
\sup _{k} E_{\varepsilon_{k}}\left(u_{k}, \Omega\right)<+\infty, \quad \sup _{k}\left\|T_{\varepsilon_{k}} u_{k}\right\|_{L^{2}\left(\mathbb{R}^{2}\right)}<+\infty
$$

$\left(u_{k}\right.$ is extended with value 0 to the whole $\left.\varepsilon \mathbb{Z}^{2}\right)$. Then there exists a subsequence (not relabelled) and a function $u_{0} \in G S B V^{2}(\Omega) \cap L^{2}(\Omega)$ such that

$$
\left.\begin{array}{l}
T_{\varepsilon_{k}} u_{k} \rightarrow u_{0} \\
S_{\varepsilon_{k}} u_{k} \rightarrow u_{0}
\end{array}\right\} \quad \text { a.e. and strongly in } L^{q}(\Omega) \text { for every } 1 \leq q<2 \text {. }
$$

Moreover, for every $k$ there exists a finite union $Z_{k}$ of squares of the mesh $\varepsilon_{k} \mathbb{Z}^{2}$, with $\left|Z_{k}\right| \rightarrow 0$, such that the functions

$$
v_{k}:= \begin{cases}S_{\varepsilon_{k}} u_{k} & \text { on } \Omega \backslash Z_{k} \\ 0 & \text { on } Z_{k}\end{cases}
$$

have the following convergence properties:

$$
\begin{aligned}
& v_{k} \rightarrow u_{0} \text { a.e. and strongly in } L^{q}(\Omega) \text { for every } 1 \leq q<2 \text {; } \\
& \nabla v_{k} \rightarrow \nabla u_{0} \quad \text { a.e. in } \Omega ; \\
& \nabla^{2} v_{k} \rightarrow \nabla^{2} u_{0} \quad \text { weakly in } L^{2}\left(\Omega ; M^{2 \times 2}\right) .
\end{aligned}
$$

Proof. Extend $u_{k}$ to $\varepsilon_{k} \mathbb{Z}^{2}$ with value zero outside $\Omega_{\varepsilon_{k}}$. By the assumptions on $\partial \Omega$, if $N_{k}=\#\left(\bar{\Omega}_{\varepsilon_{k}} \backslash \Omega_{\varepsilon_{k}}\right)$, then $N_{k}=O\left(1 / \varepsilon_{k}\right)$ (it is sufficient to consider the points $(x, y) \in \varepsilon_{k} \mathbb{Z}^{2}$ whose distance from $\partial \Omega$ does not exceed $\left.\varepsilon_{k} \sqrt{2}\right)$. Since

$$
E_{\varepsilon_{k}}\left(u_{k}, \varepsilon_{k} \mathbb{Z}^{2}\right) \leq E_{\varepsilon_{k}}\left(u_{k}, \Omega\right)+4 N_{k} \varepsilon_{k}^{2} \frac{\gamma}{\varepsilon_{k}}
$$

we deduce that the sequence $\left(E_{\varepsilon_{k}}\left(u_{k}, \varepsilon_{k} \mathbb{Z}^{2}\right)\right)_{k}$ is bounded. Let $\mathcal{Z}_{k}$ be the family of those (closed) squares $Q$ of the mesh $\varepsilon_{k} \mathbb{Z}^{2}$ such that

$$
\left|\left(\Delta_{\varepsilon_{k}}^{\alpha} u_{k}\right)(z)\right| \geq \sqrt{\gamma / \varepsilon_{k}}
$$

for at least one vertex $z$ of $Q$ and a multiindex $\alpha$ with $|\alpha|=2$. Note that

$$
E_{\varepsilon_{k}}\left(u_{k}, \varepsilon_{k} \mathbb{Z}^{2}\right) \geq \frac{1}{4} \sum_{Q \in \mathcal{Z}_{k}} \sum_{(z \text { vertex of } Q)} \sum_{|\alpha|=2} \varepsilon_{k}^{2} \psi_{\varepsilon_{k}}\left(\left(\Delta_{\varepsilon_{k}}^{\alpha} u_{k}\right)(z)\right) \geq \frac{1}{4} \sum_{Q \in \mathcal{Z}_{k}} \varepsilon_{k}^{2} \gamma / \varepsilon_{k}=\frac{1}{4}\left(\# \mathcal{Z}_{k}\right) \gamma \varepsilon_{k} .
$$

Therefore $\sup _{k}\left(\# \mathcal{Z}_{k}\right) \varepsilon_{k}<+\infty$.

For every $k$ let $Z_{k}=\bigcup \mathcal{Z}_{k}$, and

$$
v_{k}= \begin{cases}S_{\varepsilon_{k}} u_{k} & \text { on } \mathbb{R}^{2} \backslash Z_{k} \\ 0 & \text { otherwise. }\end{cases}
$$

If $B_{k}$ denotes the boundary of $Z_{k}$, then $v_{k}$ is $H^{2}$ on $\mathbb{R}^{2} \backslash B_{k}$; moreover, $v_{k} \in L^{2}(\Omega)$ by Remark 5.1. Thus, $v_{k} \in G S B V^{2}(\Omega) \cap L^{2}(\Omega)$.

On the space $G S B V^{2}(\Omega)$ consider the functional

$$
F(v)=\int_{\Omega}\left(\left|\nabla^{2} v\right|^{2}+|v|^{2}\right) \mathrm{d} x \mathrm{~d} y+\mathcal{H}^{1}\left(S_{v} \cup S_{\nabla v}\right) .
$$

We aim at proving that $\left(F\left(v_{k}\right)\right)_{k}$ is bounded. Clearly, this is true for the term $\mathcal{H}^{1}\left(S_{v_{k}} \cup S_{\nabla v_{k}}\right) \leq 4\left(\# \mathcal{Z}_{k}\right) \varepsilon_{k}$. Moreover, the sequence $\left(v_{k}\right)$ is bounded in $L^{2}(\Omega)$ as a consequence of Remark 5.1. 
Finally, by Corollary 4.7 there exists a constant $C$ such that for every $k$

$$
\begin{aligned}
\int_{\Omega}\left|\nabla^{2} v_{k}\right|^{2} \mathrm{~d} x \mathrm{~d} y & \leq \int_{\mathbb{R}^{2}}\left|\nabla^{2} v_{k}\right|^{2} \mathrm{~d} x \mathrm{~d} y=\int_{\mathbb{R}^{2} \backslash \cup \mathcal{Z}_{k}}\left|D^{2}\left(S_{\varepsilon_{k}} u_{k}\right)\right|^{2} \mathrm{~d} x \mathrm{~d} y \\
& \leq C \sum_{Q \notin \mathcal{Z}_{k}} \sum_{(z \text { vertex of } Q)} \sum_{|\alpha|=2} \varepsilon_{k}^{2}\left|\left(\Delta_{\varepsilon_{k}}^{\alpha} u_{k}\right)(z)\right|^{2} \leq 4 C E_{\varepsilon_{k}}\left(u_{k}, \varepsilon_{k} \mathbb{Z}^{2}\right) .
\end{aligned}
$$

We can conclude that $\left(v_{k}\right)$ is a sequence in $G S B V^{2}(\Omega) \cap L^{2}(\Omega)$ such that

$$
\sup _{k} F\left(v_{k}\right)<+\infty
$$

The assumptions of the compactness Theorem 8 in [19] are thus satisfied: there exists a function $u_{0} \in$ $G S B V^{2}(\Omega) \cap L^{2}(\Omega)$ such that, up to a subsequence, (5.3) holds. Since $Z_{k}$ has vanishing Lebesgue measure, the sequence $\left(S_{\varepsilon_{k}} u_{k}\right)_{k}$ is bounded in $L^{2}$, and $q<2$, this also implies the stated convergence for $\left(S_{\varepsilon_{k}} u_{k}\right)_{k}$.

Now, we have to consider the sequence $\left(T_{\varepsilon_{k}} u_{k}\right)_{k}$ of the piecewise-constant interpolations. With the letter $Q$ we will denote any square of the mesh $\varepsilon_{k} \mathbb{Z}^{2}$. For such $Q$ we can write $S_{\varepsilon_{k}} u_{k}$ as in Remark 4.4

$$
S_{\varepsilon_{k}} u_{k}(x, y)=\sum_{i, j=0}^{3} a_{i j}^{Q} \frac{\left(x-x_{Q}\right)^{i}\left(y-y_{Q}\right)^{j}}{\left(\varepsilon_{k} / 2\right)^{i+j}}
$$

Let $w_{k}$ be the piecewise-affine function which is defined by

$$
w_{k}(x, y)=a_{00}^{Q}+2 \frac{a_{10}^{Q}}{\varepsilon_{k}}\left(x-x_{Q}\right)+2 \frac{a_{01}^{Q}}{\varepsilon_{k}}\left(y-y_{Q}\right)
$$

on each square $Q \notin \mathcal{Z}_{k}$, and $w_{k}=0$ on those $Q$ in $\mathcal{Z}_{k}$. Let us show that $w_{k} \rightarrow u_{0}$ in $L^{q}$ for every $1 \leq q<2$. We have:

$$
\int_{\Omega}\left|v_{k}-w_{k}\right|^{q} \mathrm{~d} x \mathrm{~d} y \leq C \sum_{Q \notin \mathcal{Z}_{k}} \varepsilon_{k}^{2} \sum_{\substack{i, j=0 \\ i+j \geq 2}}^{3}\left|a_{i j}^{Q}\right|^{q}
$$

(here and in the sequel of the proof, $C$ denotes a positive constant suitable for the current inequality). By the boundedness of $\Omega$, the number of all the squares is of order $1 / \varepsilon_{k}^{2}$. Therefore

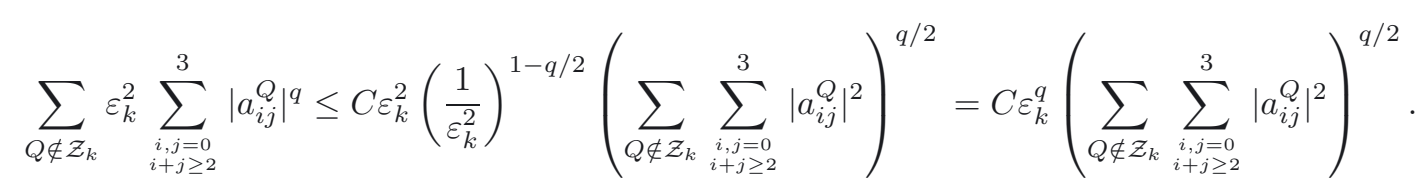

Moreover, taking Proposition 4.5 and equation (4.3) into account, we have

$$
\sum_{Q \notin \mathcal{Z}_{k}} \sum_{\substack{i, j=0 \\ i+j \geq 2}}^{3}\left|a_{i j}^{Q}\right|^{2} \leq C \sum_{Q \notin \mathcal{Z}_{k}} \sum_{|\alpha|=2} \sum_{(z \text { vertex of } Q)} \varepsilon_{k}^{4}\left|\left(\Delta_{\varepsilon}^{\alpha} u_{k}\right)(z)\right|^{2} \leq 4 C \varepsilon_{k}^{2} E_{\varepsilon_{k}}\left(u_{k}, \Omega\right) .
$$

We deduce that

$$
\sum_{Q \notin \mathcal{Z}_{k}} \varepsilon_{k}^{2} \sum_{\substack{i, j=0 \\ i+j \geq 2}}^{3}\left|a_{i j}^{Q}\right|^{q} \leq C \varepsilon_{k}^{2 q}\left(E_{\varepsilon_{k}}\left(u_{k}, \Omega\right)\right)^{q / 2} \rightarrow 0 \quad \text { as } k \rightarrow+\infty .
$$

By (5.4) we conclude that $\left\|v_{k}-w_{k}\right\|_{L^{q}} \rightarrow 0$, therefore $\left\|w_{k}-u_{0}\right\|_{L^{q}} \rightarrow 0$, too. 
Let $\bar{w}_{k}$ be the piecewise-constant function which takes the value $f_{Q} w_{k}$ (the average of $w_{k}$ on $Q$ ) on each square $Q$. We claim that $\left(\bar{w}_{k}\right)$ converges to $u_{0}$ in $L^{q}\left(\mathbb{R}^{2}\right)$ for every $1 \leq q<2$. Indeed,

$$
\begin{aligned}
\int_{\mathbb{R}^{2}}\left|\bar{w}_{k}(z)-u_{0}(z)\right|^{q} \mathrm{~d} z & =\sum_{Q} \int_{Q}\left|f_{Q} w_{k}\left(z^{\prime}\right) \mathrm{d} z^{\prime}-u_{0}(z)\right|^{q} \mathrm{~d} z \\
& \leq 2^{q-1} \sum_{Q}\left[\int_{Q}\left|f_{Q}\left(w_{k}\left(z^{\prime}\right)-u_{0}\left(z^{\prime}\right)\right) \mathrm{d} z^{\prime}\right|^{q} \mathrm{~d} z+\int_{Q}\left|f_{Q} u_{0}\left(z^{\prime}\right) \mathrm{d} z^{\prime}-u_{0}(z)\right|^{q} \mathrm{~d} z\right] \\
& \leq 2^{q-1} \sum_{Q}\left[\int_{Q} \mathrm{~d} z f_{Q}\left|w_{k}\left(z^{\prime}\right)-u_{0}\left(z^{\prime}\right)\right|^{q} \mathrm{~d} z^{\prime}+\int_{Q}\left|f_{k}(z)\right|^{q} \mathrm{~d} z\right]=: 2^{q-1}\left(I_{k}^{1}+I_{k}^{2}\right),
\end{aligned}
$$

where $f_{k}$ is the function defined a.e. on $\mathbb{R}^{2}$ by

$$
f_{k}(z)=f_{Q(z)} u_{0}\left(z^{\prime}\right) \mathrm{d} z^{\prime}-u_{0}(z)
$$

with $Q(z)$ denoting the unique open square of the mesh $\varepsilon_{k} \mathbb{Z}^{2}$ containing $z$.

Clearly

$$
I_{k}^{1}=\sum_{Q} \int_{Q}\left|w_{k}\left(z^{\prime}\right)-u_{0}\left(z^{\prime}\right)\right|^{q} \mathrm{~d} z^{\prime}=\int_{\mathbb{R}^{2}}\left|w_{k}\left(z^{\prime}\right)-u_{0}\left(z^{\prime}\right)\right|^{q} \mathrm{~d} z^{\prime} \rightarrow 0
$$

as $k \rightarrow+\infty$. As for $I_{k}^{2}$, we note that for $k$ sufficiently large $f_{k}$ vanishes outside a fixed neighborhood of $\bar{\Omega}$; moreover, $f_{k} \rightarrow 0$ a.e. on $\mathbb{R}^{2}$. Let us now look for a summable function dominating $\left|f_{k}\right|^{q}$. We have

$$
f_{Q(z)}\left|u_{0}\left(z^{\prime}\right)\right| \mathrm{d} z^{\prime} \leq \frac{1}{\varepsilon_{k}^{2}} \int_{B_{\sqrt{2} \varepsilon_{k}}(z)}\left|u_{0}\left(z^{\prime}\right)\right| \mathrm{d} z^{\prime} \leq 2 \pi M\left(u_{0}\right)(z),
$$

where $M\left(u_{0}\right)$ denotes the Hardy-Littlewood maximal function of $u_{0}$. Since $u_{0} \in L^{2}\left(\mathbb{R}^{2}\right)$, it follows that $M\left(u_{0}\right) \in$ $L^{2}\left(\mathbb{R}^{2}\right)$, too. Since $f_{k}$ vanishes outside a compact set, the function $\left(2 \pi M\left(u_{0}\right)+\left|u_{0}\right|\right)^{q}$ yields a summable bound for $\left|f_{k}\right|^{q}$. By the dominated convergence theorem we get

$$
I_{k}^{2}=\int_{\mathbb{R}^{2}}\left|f_{k}(z)\right|^{q} \mathrm{~d} z \rightarrow 0 \quad \text { as } k \rightarrow+\infty .
$$

We conclude that $\bar{w}_{k} \rightarrow u_{0}$ in $L^{q}(\Omega)$.

Clearly for every $Q \notin \mathcal{Z}_{k}$ we have $\bar{w}_{k}=a_{00}^{Q}$ on $Q$; hence,

$$
\begin{aligned}
\sum_{Q \notin \mathcal{Z}_{k}} \int_{Q}\left|\bar{w}_{k}(z)-w_{k}(z)\right|^{q} \mathrm{~d} z & =\sum_{Q \notin \mathcal{Z}_{k}} \int_{Q}\left|2 a_{10}^{Q} \frac{x-x_{Q}}{\varepsilon_{k}}+2 a_{01}^{Q} \frac{y-y_{Q}}{\varepsilon_{k}}\right|^{q} \mathrm{~d} x \mathrm{~d} y \\
& \geq \sum_{Q \notin \mathcal{Z}_{k}} \int_{A_{Q}}\left[\left(2 a_{10}^{Q} \frac{x-x_{Q}}{\varepsilon_{k}}\right)^{q}+\left(2 a_{01}^{Q} \frac{y-y_{Q}}{\varepsilon_{k}}\right)^{q}\right] \mathrm{d} x \mathrm{~d} y
\end{aligned}
$$

where $A_{Q}=\left\{(x, y) \in Q: a_{10}^{Q}\left(x-x_{Q}\right) \geq 0, a_{01}^{Q}\left(y-y_{Q}\right) \geq 0\right\}$. It turns out that

$$
\int_{A_{Q}}\left[\left(2 a_{10}^{Q} \frac{x-x_{Q}}{\varepsilon_{k}}\right)^{q}+\left(2 a_{01}^{Q} \frac{y-y_{Q}}{\varepsilon_{k}}\right)^{q}\right] \mathrm{d} x \mathrm{~d} y=\frac{1}{q+1}\left(\frac{\varepsilon_{k}}{2}\right)^{2}\left(\left|a_{10}^{Q}\right|^{q}+\left|a_{01}^{Q}\right|^{q}\right) .
$$

Thus, the convergence of both $\left(w_{k}\right)_{k}$ and $\left(\bar{w}_{k}\right)_{k}$ to $u_{0}$ in $L^{q}$ implies that

$$
\sum_{Q \notin \mathcal{Z}_{k}} \sum_{i+j=1} \varepsilon_{k}^{2}\left|a_{i j}^{Q}\right|^{q} \rightarrow 0
$$


We now prove the convergence of $\left(T_{\varepsilon_{k}} u_{k}\right)_{k}$. We have:

$$
\left\|T_{\varepsilon_{k}} u_{k}-\bar{w}_{k}\right\|_{L^{q}(\Omega)}^{q}=\sum_{Q \in \mathcal{Z}_{k}} \int_{Q}\left|T_{\varepsilon_{k}} u_{k}\right|^{q} \mathrm{~d} x \mathrm{~d} y+\sum_{Q \notin \mathcal{Z}_{k}} \int_{Q}\left|T_{\varepsilon_{k}} u_{k}-\bar{w}_{k}\right|^{q} \mathrm{~d} x \mathrm{~d} y=: J_{k}^{1}+J_{k}^{2} .
$$

Since $Z_{k}=\bigcup \mathcal{Z}_{k}$ has vanishing Lebesgue measure, and $T_{\varepsilon_{k}} u_{k}$ are equibounded in $L^{2}, J_{k}^{1} \rightarrow 0$. As to the second term, given a square $Q$ of the mesh, let $\bar{z}=(\bar{x}, \bar{y})$ be the left lower corner of $Q$; then $T_{\varepsilon_{k}} u_{k}=u_{k}(\bar{z})=S_{\varepsilon_{k}} u_{k}(\bar{z})$ on $Q$ and

$$
\begin{aligned}
J_{k}^{2} & =\sum_{Q \notin \mathcal{Z}_{k}} \int_{Q}\left|S_{\varepsilon_{k}} u_{k}(\bar{z})-a_{00}^{Q}\right|^{q} \mathrm{~d} x \mathrm{~d} y \\
& =\sum_{Q \notin \mathcal{Z}_{k}} \varepsilon_{k}^{2}\left|\sum_{i+j \geq 1} a_{i j}^{Q} \frac{\left(\bar{x}-x_{Q}\right)^{i}\left(\bar{y}-y_{Q}\right)^{j}}{\left(\varepsilon_{k} / 2\right)^{i+j}}\right|^{q} \leq C \sum_{Q \notin \mathcal{Z}_{k}} \varepsilon_{k}^{2} \sum_{i+j \geq 1}\left|a_{i j}^{Q}\right|^{q} .
\end{aligned}
$$

It is now enough to apply (5.6), for the terms $a_{i j}^{Q}$ with $i+j=1$ and (5.5) when $i+j \geq 2$, in order to conclude that $\left\|T_{\varepsilon_{k}} u_{k}-\bar{w}_{k}\right\|_{L^{q}(\Omega)} \rightarrow 0$.

\section{Estimates for the $\Gamma$-Limit}

In this section we obtain upper and lower estimates for the $\Gamma$-limit of the functionals $E_{\varepsilon}$. For the lower bound we reduce the problem to a one-dimensional setting through a slicing technique. Thus, the first and third term in definition (3.2) can be managed by means of Theorem 3.2, while for the mixed-derivatives term we have to exploit the convergence of the gradients guaranteed by the compactness theorem of the previous section, and then apply a convergence result for first-order functionals (see Chambolle $[25,26]$ ).

Theorem 6.1 (lower estimate). Let $\Omega$ be a bounded open subset of $\mathbb{R}^{2}$. Let $\left(u_{k}\right)_{k}$ and $u_{0}$ be as in Theorem 5.2. Then

$$
\begin{aligned}
& \liminf _{k \rightarrow+\infty} E_{\varepsilon_{k}}\left(u_{\varepsilon_{k}}, \Omega\right) \geq \int_{\Omega}\left|\nabla^{2} u_{0}(x, y)\right|^{2} \mathrm{~d} x \mathrm{~d} y+2 \gamma \int_{S_{u_{0}}}\|\nu\|_{1} \mathrm{~d} \mathcal{H}^{1}+\gamma \int_{S_{\nabla_{x} u_{0}} \backslash S_{u_{0}}}\left|\nu_{1}\right| \mathrm{d} \mathcal{H}^{1}+\gamma \int_{S_{\nabla_{y} u_{0} \backslash S_{u_{0}}}}\left|\nu_{2}\right| \mathrm{d} \mathcal{H}^{1} \\
& +2 \gamma \int_{S_{\nabla_{y} u_{0}} \backslash S_{\nabla_{x} u_{0}}}\left|\nu_{1}\right| \mathrm{d} \mathcal{H}^{1}+2 \gamma \int_{S_{\nabla_{x} u_{0}} \backslash S_{\nabla_{y} u_{0}}}\left|\nu_{2}\right| \mathrm{d} \mathcal{H}^{1}+2 \gamma \int_{S_{\nabla_{y} u_{0}} \cap S_{\nabla_{x} u_{0}}}\|\nu\|_{\infty} \mathrm{d} \mathcal{H}^{1},
\end{aligned}
$$

where $\|\nu\|_{1}=\left|\nu_{1}\right|+\left|\nu_{2}\right|$ and $\|\nu\|_{\infty}=\max \left(\left|\nu_{1}\right|,\left|\nu_{2}\right|\right)$.

Proof. For notational convenience, in the sequel we will simply write $\varepsilon$ instead of $\varepsilon_{k}$. We can assume that $E_{\varepsilon}\left(u_{\varepsilon}, \Omega\right)$ converge for $\varepsilon \rightarrow 0$, so that the possible extraction of subsequences does not affect the estimate of the lower limit.

Let $\Omega^{\prime} \subset \subset \Omega$ be fixed. If $\varepsilon>0$ is sufficiently small, then

$$
\sum_{(x, y) \in \Omega_{\varepsilon}} \varepsilon^{2} \psi_{\varepsilon}\left(\Delta_{\varepsilon}^{(2,0)} u_{\varepsilon}(x, y)\right) \geq \int_{\mathbb{R}} \sum_{x \in\left(\Omega^{\prime}\right)^{y} \cap \varepsilon \mathbb{Z}} \varepsilon \psi_{\varepsilon}\left(\frac{\hat{u}_{\varepsilon}^{y}(x+\varepsilon)+\hat{u}_{\varepsilon}^{y}(x-\varepsilon)-2 \hat{u}_{\varepsilon}^{y}(x)}{\varepsilon^{2}}\right) \mathrm{d} y,
$$

where $\left(\Omega^{\prime}\right)^{y}$ and $\hat{u}_{\varepsilon}^{y}$ denote the one-dimensional sections of $\Omega^{\prime}$ and $\hat{u}_{\varepsilon}$, respectively, introduced in (2.1). Since (up to a subsequence) $\hat{u}_{\varepsilon}^{y} \rightarrow u_{0}^{y}$ in $L^{1}\left(\Omega^{y}\right)$ for a.e. $y \in \mathbb{R}$, we are in a position to apply Theorem 3.2 to each connected component of $\left(\Omega^{\prime}\right)^{y}$. Thus $u_{0}^{y}$ is piecewise- $H^{2}$ on $\left(\Omega^{\prime}\right)^{y}$ and

$$
\begin{aligned}
\liminf _{\varepsilon \rightarrow 0} & \sum_{(x, y) \in \Omega_{\varepsilon}} \varepsilon^{2} \psi_{\varepsilon}\left(\Delta_{\varepsilon}^{(2,0)} u_{\varepsilon}(x, y)\right) \\
\geq & \int_{\mathbb{R}}\left(\int_{\left(\Omega^{\prime}\right)^{y}}\left|\left(u_{0}^{y}\right)^{\prime \prime}(x)\right|^{2} \mathrm{~d} x+\gamma \#\left[\left(S_{\left(u_{0}^{y}\right)^{\prime}} \backslash S_{u_{0}^{y}}\right) \cap \Omega^{\prime}\right]+2 \gamma \#\left[S_{u_{0}^{y}} \cap \Omega^{\prime}\right]\right) \mathrm{d} y .
\end{aligned}
$$


Since $u_{0} \in G S B V^{2}(\Omega)$ (Thm. 5.2), by the arbitrariness of $\Omega^{\prime}$, the same inequality holds with $\Omega$ in place of $\Omega^{\prime}$. On account of Theorem 2.1 we have

$$
\int_{\mathbb{R}} \int_{\left(\Omega^{\prime}\right)^{y}}\left|\left(u_{0}^{y}\right)^{\prime \prime}(x)\right|^{2} \mathrm{~d} x \mathrm{~d} y=\int_{\Omega}\left|\nabla_{x x}^{2} u_{0}(x, y)\right|^{2} \mathrm{~d} x \mathrm{~d} y .
$$

Moreover, Theorem 2.1 also yields that, for a.e. $y \in \mathbb{R}$ with $\Omega^{y} \neq \emptyset$,

$$
S_{u_{0}^{y}}=\left(S_{u_{0}}\right)^{y}, \quad\left(u_{0}^{y}\right)^{\prime}=\nabla_{x} u_{0}(\cdot, y)=\left(\nabla_{x} u_{0}\right)^{y} \quad \text { a.e. on } \Omega^{y} .
$$

Therefore we have $S_{\left(u_{0}^{y}\right)^{\prime}}=\left(S_{\nabla_{x} u_{0}}\right)^{y}$, too; thus

$$
S_{\left(u_{0}^{y}\right)^{\prime}} \backslash S_{u_{0}^{y}}=\left(S_{\nabla_{x} u_{0}}\right)^{y} \backslash\left(S_{u_{0}}\right)^{y}=\left(S_{\nabla_{x} u_{0}} \backslash S_{u_{0}}\right)^{y} .
$$

By the coarea formula

$$
\int_{\mathbb{R}} \#\left(E^{y}\right) \mathrm{d} y=\int_{E}\left|\nu_{1}\right| \mathrm{d} \mathcal{H}^{1}
$$

whenever $E \subseteq \mathbb{R}^{2}$ is a $\mathcal{H}^{1}$-rectifiable set with $\nu=\left(\nu_{1}, \nu_{2}\right)$ as unit normal field; hence,

$$
\int_{\mathbb{R}} \# S_{u_{0}^{y}} \mathrm{~d} y=\int_{S_{u_{0}}}\left|\nu_{1}\right| \mathrm{d} \mathcal{H}^{1}, \quad \int_{\mathbb{R}} \#\left(S_{\left(u_{0}^{y}\right)^{\prime}} \backslash S_{u_{0}^{y}}\right) \mathrm{d} y=\int_{S_{\nabla_{x} u_{0}} \backslash S_{u_{0}}}\left|\nu_{1}\right| \mathrm{d} \mathcal{H}^{1} .
$$

We conclude that

$$
\liminf _{\varepsilon \rightarrow 0} \sum_{(x, y) \in \Omega_{\varepsilon}} \varepsilon^{2} \psi_{\varepsilon}\left(\Delta_{\varepsilon}^{(2,0)} u_{\varepsilon}(x, y)\right) \geq \int_{\Omega}\left|\nabla_{x x}^{2} u_{0}(x, y)\right|^{2} \mathrm{~d} x \mathrm{~d} y+2 \gamma \int_{S_{u_{0}}}\left|\nu_{1}\right| \mathrm{d} \mathcal{H}^{1}+\gamma \int_{S_{\nabla_{x} u_{0} \backslash S_{u_{0}}}}\left|\nu_{1}\right| \mathrm{d} \mathcal{H}^{1} .
$$

An analogous estimate for the term involving $\Delta_{\varepsilon}^{(0,2)}$ clearly holds.

We now turn to the mixed-derivatives term involving $\Delta_{\varepsilon}^{(1,1)}$. If $\varepsilon>0$ is sufficiently small, then for every $(x, y) \in \Omega^{\prime} \cap \varepsilon \mathbb{Z}^{2}$ each square of the mesh with a vertex in $(x, y)$ is contained in $\Omega$. Therefore

$$
\Delta_{\varepsilon}^{(1,1)} u_{\varepsilon}(x, y)=\frac{1}{\varepsilon}\left[\Delta_{\varepsilon}^{(0,1)} u_{\varepsilon}(x+\varepsilon, y)-\Delta_{\varepsilon}^{(0,1)} u_{\varepsilon}(x, y)\right]=\frac{1}{\varepsilon}\left[D_{y} S_{\varepsilon} u_{\varepsilon}(x+\varepsilon, y)-D_{y} S_{\varepsilon} u_{\varepsilon}(x, y)\right] .
$$

We now introduce the function $s_{\varepsilon}=T_{\varepsilon}\left(D_{y} S_{\varepsilon} u_{\varepsilon}\right)$, i.e. the piecewise constant extension of $\left.\left(D_{y} S_{\varepsilon} u_{\varepsilon}\right)\right|_{\varepsilon \mathbb{Z}^{2}}$ according to (5.1). Since $\Omega^{\prime} \subset \subset \Omega$, we have:

$$
\sum_{(x, y) \in \Omega_{\varepsilon}} \varepsilon^{2} \psi_{\varepsilon}\left(\Delta_{\varepsilon}^{(1,1)} u_{\varepsilon}(x, y)\right) \geq \int_{\mathbb{R}} \sum_{x \in\left(\Omega^{\prime}\right)^{y} \cap \varepsilon \mathbb{Z}} \varepsilon \psi_{\varepsilon}\left(\frac{s_{\varepsilon}^{y}(x+\varepsilon)-s_{\varepsilon}^{y}(x)}{\varepsilon}\right) \mathrm{d} y .
$$

We would like to apply Chambolle's one-dimensional convergence result (see [25,26]) to the integrand function. To this end we need the pointwise convergence of $s_{\varepsilon}$.

Note that, for any given $N \in \mathbb{N}$ there exists a constant $C$ such that if $p(x, y)$ is a polynomial whose degree does not exceed $N$ and if $p(0,0)=0$, then

$$
\int_{[0,1]^{2}}|p(x, y)|^{2} \mathrm{~d} x \mathrm{~d} y \leq C \int_{[0,1]^{2}}|D p(x, y)|^{2} \mathrm{~d} x \mathrm{~d} y
$$

(indeed, $\int_{[0,1]^{2}}|p(x, y)|^{2} \mathrm{~d} x \mathrm{~d} y / \int_{[0,1]^{2}}|D p(x, y)|^{2} \mathrm{~d} x \mathrm{~d} y$ is a 0-homogeneous function of the coefficients $a=\left(a_{i j}\right)$ of $p(x, y)=\sum_{i, j} a_{i j} x^{i} y^{j}$, defined for every $\left.a \neq 0\right)$. By translating and rescaling we deduce that there exists 
a constant $C$ such that for every $\left(x_{0}, y_{0}\right) \in \mathbb{R}^{2}$, if $Q_{\varepsilon}\left(x_{0}, y_{0}\right)=\left[x_{0}, x_{0}+\varepsilon\right) \times\left[y_{0}, y_{0}+\varepsilon\right)$ then

$$
\int_{Q_{\varepsilon}\left(x_{0}, y_{0}\right)}\left|p(x, y)-p\left(x_{0}, y_{0}\right)\right|^{2} \mathrm{~d} x \mathrm{~d} y \leq C \varepsilon^{2} \int_{Q_{\varepsilon}\left(x_{0}, y_{0}\right)}|D p(x, y)|^{2} \mathrm{~d} x \mathrm{~d} y,
$$

whenever $p(x, y)$ is a polynomial whose degree does not exceed $N$.

Apply this inequality to the polynomial $p=D_{y} S_{\varepsilon} u_{\varepsilon}$ on every square $Q_{\varepsilon}\left(x_{0}, y_{0}\right)$ (of the mesh $\varepsilon \mathbb{Z}^{2}$ ) covering $\Omega^{\prime}$ (when $\varepsilon$ is sufficiently small $S_{\varepsilon} u_{\varepsilon}$ is defined on each of these squares). Note that for every $(x, y) \in Q_{\varepsilon}\left(x_{0}, y_{0}\right)$

$$
s_{\varepsilon}(x, y)=D_{y} S_{\varepsilon} u_{\varepsilon}\left(x_{0}, y_{0}\right)=p\left(x_{0}, y_{0}\right) .
$$

Therefore

$$
\int_{Q_{\varepsilon}\left(x_{0}, y_{0}\right)}\left|D_{y} S_{\varepsilon} u_{\varepsilon}(x, y)-s_{\varepsilon}(x, y)\right|^{2} \mathrm{~d} x \mathrm{~d} y \leq C \varepsilon^{2} \int_{Q_{\varepsilon}\left(x_{0}, y_{0}\right)}\left|D^{2} S_{\varepsilon} u_{\varepsilon}(x, y)\right|^{2} \mathrm{~d} x \mathrm{~d} y .
$$

Let $v_{\varepsilon}$ be as in Theorem 5.2, i.e.: $v_{\varepsilon}=0$ on those squares where $\left|\left(\Delta_{\varepsilon}^{\alpha} u_{\varepsilon}\right)(z)\right| \geq \sqrt{\gamma / \varepsilon}$ for at least one vertex $z$ of $Q$ and a multiindex $\alpha$ with $|\alpha|=2$; and $v_{\varepsilon}=S_{\varepsilon} u_{\varepsilon}$ outside the union $Z_{\varepsilon}$ of these squares. Then, by (6.3),

$$
\int_{\Omega^{\prime}}\left|\nabla_{y} v_{\varepsilon}-s_{\varepsilon} \chi_{\varepsilon}\right|^{2} \mathrm{~d} x \mathrm{~d} y \leq C \varepsilon^{2} \sum_{Q_{\varepsilon}} \int_{Q_{\varepsilon}}\left|D^{2} S_{\varepsilon} u_{\varepsilon}(x, y)\right|^{2} \mathrm{~d} x \mathrm{~d} y
$$

where $\chi_{\varepsilon}$ denotes the function which takes the values 0 on $Z_{\varepsilon}$ and 1 otherwise, and the sum on the right-hand side ranges over all the squares covering $\Omega^{\prime}$ but outside $Z_{\varepsilon}$. By Corollary 4.7 , and the definition of $Z_{\varepsilon}$ we can estimate this sum by $E_{\varepsilon}\left(u_{\varepsilon}, \Omega\right)$, which is bounded by assumption. Since $\nabla_{y} v_{\varepsilon} \rightarrow \nabla_{y} u_{0}$ a.e. by Theorem 5.2 , we deduce that (up to a subsequence), $s_{\varepsilon} \chi_{\varepsilon} \rightarrow \nabla_{y} u_{0}$ a.e. in $\Omega^{\prime}$. Since $\left|Z_{\varepsilon}\right| \rightarrow 0$, possibly extracting a further subsequence, we get

$$
s_{\varepsilon} \rightarrow \nabla_{y} u_{0} \quad \text { a.e. in } \Omega^{\prime} .
$$

We now recall Chambolle's convergence results $[25,26]$ : let $(a, b)$ be a given open interval, and let $\left(w_{\varepsilon}\right)$ be a sequence of real-valued functions on $(a, b) \cap \varepsilon \mathbb{Z}$, extended with value 0 in $\varepsilon \mathbb{Z} \backslash(a, b)$. Assume $w_{\varepsilon}$ defined, as a piecewise constant function, all over $(a, b)$ by $w_{\varepsilon}(x)=w_{\varepsilon}(\varepsilon\lfloor x / \varepsilon\rfloor)$. If $\left(w_{\varepsilon}\right)$ converges a.e. in $(a, b)$ to a $S B V$ function $w$, then

$$
\liminf _{\varepsilon \rightarrow 0} \sum_{\substack{x \in \varepsilon \mathbb{Z} \\ x, x+\varepsilon \in(a, b)}} \varepsilon \psi_{\varepsilon}\left(\frac{w_{\varepsilon}(x+\varepsilon)-w_{\varepsilon}(x)}{\varepsilon}\right) \geq \int_{(a, b) \backslash S_{w}}\left|w^{\prime}\right|^{2} \mathrm{~d} x+\gamma \# S_{w} .
$$

Let $M>0$ be fixed. We are in a position to apply the inequality above for a.e. $y \in \mathbb{R}$ on each connected component of $\left(\Omega^{\prime}\right)^{y}$ (to be precise, for each of them we have to consider a sequence of compact intervals invading it), and with

$$
w_{\varepsilon}=w_{\varepsilon}^{y}:=(-M) \vee s_{\varepsilon}(\cdot, y) \wedge M, \quad w=w^{y}:=(-M) \vee \nabla_{y} u_{0}(\cdot, y) \wedge M .
$$

Take now (6.2) into account; since the first-order quotients decrease by truncation we get

$$
\liminf _{\varepsilon \rightarrow 0} \sum_{(x, y) \in \Omega_{\varepsilon}} \varepsilon^{2} \psi_{\varepsilon}\left(\Delta_{\varepsilon}^{(1,1)} u_{\varepsilon}(x, y)\right) \geq \int_{\mathbb{R}}\left(\int_{\Omega^{y}}\left|\left(w^{y}\right)^{\prime}\right|^{2} \mathrm{~d} x+\gamma \# S_{w^{y}}\right) \mathrm{d} y .
$$

By letting $M$ tend to $+\infty$ we conclude that

$$
\liminf _{\varepsilon \rightarrow 0} \sum_{(x, y) \in \Omega_{\varepsilon}} \varepsilon^{2} \psi_{\varepsilon}\left(\Delta_{\varepsilon}^{(1,1)} u_{\varepsilon}(x, y)\right) \geq \int_{\mathbb{R}}\left(\int_{\Omega^{y}}\left|\left[\left(\nabla_{y} u_{0}\right)^{y}\right]^{\prime}\right|^{2} \mathrm{~d} x+\gamma \# S_{\left(\nabla_{y} u_{0}\right)^{y}}\right) \mathrm{d} y .
$$


As before this implies that

$$
\liminf _{\varepsilon \rightarrow 0} \sum_{(x, y) \in \Omega_{\varepsilon}} \varepsilon^{2} \psi_{\varepsilon}\left(\Delta_{\varepsilon}^{(1,1)} u_{\varepsilon}(x, y)\right) \geq \int_{\Omega}\left|\nabla_{x y}^{2} u_{0}(x, y)\right|^{2} \mathrm{~d} x \mathrm{~d} y+\gamma \int_{S_{\nabla_{y} u_{0}}}\left|\nu_{1}\right| \mathrm{d} \mathcal{H}^{1}
$$

An analogous estimate holds with the roles of $x$ and $y$ interchanged:

$$
\liminf _{\varepsilon \rightarrow 0} \sum_{(x, y) \in \Omega_{\varepsilon}} \varepsilon^{2} \psi_{\varepsilon}\left(\Delta_{\varepsilon}^{(1,1)} u_{\varepsilon}(x, y)\right) \geq \int_{\Omega}\left|\nabla_{x y}^{2} u_{0}(x, y)\right|^{2} \mathrm{~d} x \mathrm{~d} y+\gamma \int_{S_{\nabla_{x} u_{0}}}\left|\nu_{2}\right| \mathrm{d} \mathcal{H}^{1} .
$$

We now consider the supremum (in a measure theoretic sense) of these two lower bounds. To this end we have to 'localize' the estimates above by applying the argument with $\Omega$ replaced by any open subset $A \subseteq \Omega$; if we set

$$
G(A)=\liminf _{\varepsilon \rightarrow 0} \sum_{(x, y) \in A_{\varepsilon}} \varepsilon^{2} \psi_{\varepsilon}\left(\Delta_{\varepsilon}^{(1,1)} u_{\varepsilon}(x, y)\right)-\int_{A}\left|\nabla_{x y}^{2} u_{0}(x, y)\right|^{2} \mathrm{~d} x \mathrm{~d} y,
$$

then

$$
G(A) \geq \int_{A}\left(\psi+\psi_{i}\right) \mathrm{d} \mathcal{H}^{1}, \quad i=1,2
$$

where

$$
\psi=\left|\nu_{1}\right| 1_{S_{\nabla_{y} u_{0}} \backslash S_{\nabla_{x} u_{0}}}+\left|\nu_{2}\right| 1_{S_{\nabla_{x} u_{0}} \backslash S_{\nabla_{y} u_{0}}}, \quad \psi_{i}=\left|\nu_{i}\right| 1_{S_{\nabla_{y} u_{0}} \cap S_{\nabla_{x} u_{0}}} .
$$

A standard measure-theoretical result (see e.g. Thm. 1.16 in [12]) yields

$$
G(A) \geq \int_{A}\left(\psi+\max \left(\psi_{1}, \psi_{2}\right)\right) \mathrm{d} \mathcal{H}^{1} .
$$

In particular we take $A=\Omega$. This inequality, together with (6.1) and the analogous one for the term involving $\Delta_{\varepsilon}^{(0,2)}$, finally results in the stated bound for the lower limit.

We now turn to the upper estimate, which relies on a construction close to the discontinuity points of $u$ and $\nabla u$. This can be compared with the one in [6], where an optimal interpolation is constructed on a tubular neighbourhood of the jump set of $u$. In our case we simply extend the function $u$ to 0 in the same neighbourhood. As in [6], since we cannot use this construction for the jump set of $\nabla u$, this argument may be sharp only when the energy on 'creases' is determined by that on 'jumps' (i.e. when $\alpha=2 \beta$ in (1.2)).

Theorem 6.2 (upper estimate). Let $\Omega$ be a star-shaped bounded open subset of $\mathbb{R}^{2}$, and let $u_{0} \in G S B V^{2}(\Omega)$ $\cap L^{2}(\Omega)$. Then for all sequences $\varepsilon_{k} \rightarrow 0$ there exists a sequence $u_{\varepsilon_{k}} \rightarrow u_{0} L^{2}$ (i.e. $T_{\varepsilon_{k}} u_{\varepsilon_{k}} \rightarrow u_{0}$ in $L^{2}$ ) such that

$$
\limsup _{k \rightarrow+\infty} E_{\varepsilon_{k}}\left(u_{\varepsilon_{k}}, \Omega\right) \leq \int_{\Omega}\left|\nabla^{2} u_{0}\right|^{2} \mathrm{~d} x \mathrm{~d} y+24 \sqrt{2} \gamma \mathcal{H}^{1}\left(S_{u_{0}} \cup S_{\nabla u_{0}}\right) .
$$

Proof. For notational convenience we will drop the index $k$ from sequences.

The idea is to define $u_{\varepsilon}$ as the discretization of $u_{0} \chi_{\Omega \backslash U_{\varepsilon}}$, where $U_{\varepsilon}$ is a suitable tubular neighbourhood of $S_{u_{0}} \cup S_{\nabla u_{0}}$. The singular set of $u_{\varepsilon}$ is thus related to $\partial U_{\varepsilon}$, and can be estimated in term of $\mathcal{H}^{1}\left(S_{u_{0}} \cup S_{\nabla u_{0}}\right)$ under the additional assumption that the 1-dimensional Hausdorff measure of $S_{u_{0}} \cup S_{\nabla u_{0}}$ coincides with its Minkowski content (see (6.6)). In order to remove this assumption, $u_{0}$ will then be approximated by a regularizing sequence of minimizers of suitable minimum problems: since the regularity of the singular sets of these minimizers is only guaranteed on the compact subsets of the domain, we will need the technical assumption that $\Omega$ is star-shaped, thus allowing a simple dilation argument which extends the functions to a domain $\Omega^{\prime}$ which strictly contains $\Omega$ (see (6.5)). 
According to the line of proof sketched above, we first deal with the case when $u_{0}$ is defined on some $\Omega^{\prime}$ with $\Omega \subset \subset \Omega^{\prime}$, and is such that, having set $K^{\prime}=\left(S_{u_{0}} \cup S_{\nabla u_{0}}\right) \cap \Omega^{\prime}$ :

$$
u_{0} \in H^{2}\left(\Omega^{\prime} \backslash \bar{K}^{\prime}\right) \cap C^{2}\left(\Omega^{\prime} \backslash \bar{K}^{\prime}\right) .
$$

Moreover, we require that if $\Omega^{\prime \prime}$ is an open set with $\Omega \subset \subset \Omega^{\prime \prime} \subset \subset \Omega^{\prime}$, then the set $K^{\prime \prime}=\left(S_{u_{0}} \cup S_{\nabla u_{0}}\right) \cap \Omega^{\prime \prime}$ has the property that

$$
\mathcal{H}^{1}\left(K^{\prime \prime}\right)=\mathcal{M}\left(K^{\prime \prime}\right)<+\infty
$$

where

$$
\mathcal{M}\left(K^{\prime \prime}\right):=\lim _{\rho \rightarrow 0} \frac{\left|\left\{x \in \Omega^{\prime}: \operatorname{dist}\left(x, K^{\prime \prime}\right)<\rho\right\}\right|}{2 \rho}
$$

denotes the Minkowski content of $K^{\prime \prime}$, and |.| stands for the Lebesgue measure.

Let us look for a suitable radius for the tubular neighborhood $U_{\varepsilon}$ of $K^{\prime \prime}$. With fixed $\rho, \delta>0$, with $\delta<\rho$, by the coarea formula, we can write

$$
\begin{aligned}
\int_{\delta+\frac{3}{2} \varepsilon \sqrt{2}}^{\rho-\frac{3}{2} \varepsilon \sqrt{2}}\left|\left\{x \in \Omega^{\prime}:\left|\operatorname{dist}\left(x, K^{\prime \prime}\right)-t\right| \leq \frac{3}{2} \varepsilon \sqrt{2}\right\}\right| \mathrm{d} t & =\int_{\delta+\frac{3}{2} \varepsilon \sqrt{2}}^{\rho-\frac{3}{2} \varepsilon \sqrt{2}} \int_{t-\frac{3}{2} \varepsilon \sqrt{2}}^{t+\frac{3}{2} \varepsilon \sqrt{2}} \mathcal{H}^{1}\left(\left\{x \in \Omega^{\prime}: \operatorname{dist}\left(x, K^{\prime \prime}\right)=s\right\}\right) \mathrm{d} s \mathrm{~d} t \\
& \leq 3 \varepsilon \sqrt{2} \int_{\delta}^{\rho} \mathcal{H}^{1}\left(\left\{x \in \Omega^{\prime}: \operatorname{dist}\left(x, K^{\prime \prime}\right)=s\right\}\right) \mathrm{d} s \\
& \leq 3 \varepsilon \sqrt{2}\left|\left\{x \in \Omega^{\prime}: \operatorname{dist}\left(x, K^{\prime \prime}\right)<\rho\right\}\right| \\
& \leq 6 \rho \varepsilon \sqrt{2}\left(\mathcal{H}^{1}\left(K^{\prime \prime}\right)+o_{\rho}(1)\right)
\end{aligned}
$$

hence we can find $\delta<t^{\varepsilon}<\rho$ such that, having set

$$
D^{\varepsilon}=\left\{x \in \Omega^{\prime}: t^{\varepsilon}-\frac{3}{2} \varepsilon \sqrt{2} \leq \operatorname{dist}\left(x, K^{\prime \prime}\right) \leq t^{\varepsilon}+\frac{3}{2} \varepsilon \sqrt{2}\right\}
$$

we have

$$
\left|D^{\varepsilon}\right| \leq \frac{\rho}{\rho-\delta-3 \varepsilon \sqrt{2}} 6 \sqrt{2} \varepsilon\left(\mathcal{H}^{1}\left(K^{\prime \prime}\right)+o_{\rho}(1)\right) .
$$

We notice that: (i) the shrinking to 0 of the radius $t^{\varepsilon}$ will be obtained as $\rho \rightarrow 0$; (ii) the radius $\frac{3}{2} \varepsilon \sqrt{2}$ is chosen in order to estimate the sum $I_{\varepsilon}^{2}$ below; (iii) the positive radius $\delta$ allows the argument for the estimate of $I_{\varepsilon}^{1}$ below.

We define $u_{\varepsilon}^{\rho, \delta}$ as the discretization of $u_{0} \chi_{\Omega \backslash U_{\varepsilon}}\left(\right.$ i.e. $\left.u_{\varepsilon}^{\rho, \delta}=T_{\varepsilon_{k}}\left(u_{0} \chi_{\Omega \backslash U_{\varepsilon}}\right)\right)$, where $U_{\varepsilon}=\left\{x \in \Omega^{\prime}: \operatorname{dist}\left(x, K^{\prime \prime}\right)<\right.$ $\left.t^{\varepsilon}\right\}$. We can write

$$
E_{\varepsilon}\left(u_{\varepsilon}^{\rho, \delta}, \Omega\right)=\sum_{z \in \Omega_{\varepsilon}} \varepsilon^{2}\left(\psi_{\varepsilon}\left(\Delta_{\varepsilon}^{(2,0)} u_{\varepsilon}^{\rho, \delta}(z)\right)+2 \psi_{\varepsilon}\left(\Delta_{\varepsilon}^{(1,1)} u_{\varepsilon}^{\rho, \delta}(z)\right)+\psi_{\varepsilon}\left(\Delta_{\varepsilon}^{(0,2)} u_{\varepsilon}^{\rho, \delta}(z)\right)\right)=I_{\varepsilon}^{1}+I_{\varepsilon}^{2}+I_{\varepsilon}^{3},
$$

where

- $I_{\varepsilon}^{1}$ is the sum on all $z \in \Omega_{\varepsilon}$ with $\operatorname{dist}(z, K)>t^{\varepsilon}+\varepsilon \sqrt{2}$,

- $I_{\varepsilon}^{2}$ is the sum on all $z \in \Omega_{\varepsilon}$ with $t^{\varepsilon}-\varepsilon \sqrt{2} \leq \operatorname{dist}(z, K) \leq t^{\varepsilon}+\varepsilon \sqrt{2}$,

- $I_{\varepsilon}^{3}$ is the sum on all $z \in \Omega_{\varepsilon}$ with $\operatorname{dist}(z, K)<t^{\varepsilon}-\varepsilon \sqrt{2}$.

Since $u_{0} \in H^{2}\left(\Omega^{\prime} \backslash \bar{K}^{\prime}\right) \cap C^{2}\left(\Omega^{\prime} \backslash \bar{K}^{\prime}\right)$, it turns out that $\left|\nabla^{2} u_{0}\right|^{2}$ is continuous on a neighborhood of $\bar{G}$, where $G=\Omega \backslash\left\{x \in \Omega^{\prime}: \operatorname{dist}\left(x, K^{\prime \prime}\right) \leq \delta\right\}$. Moreover, if $z \in \Omega_{\varepsilon}$ is a point of the sum $I_{\varepsilon}^{1}$, then each of the squares of the mesh with a vertex in $z$ is contained in $G$. Therefore it is easy to see that:

$$
\limsup _{\varepsilon \rightarrow 0} I_{\varepsilon}^{1} \leq \int_{\Omega \backslash\{x: \operatorname{dist}(x, K) \leq \delta\}}\left|\nabla^{2} u_{0}\right|^{2} \mathrm{~d} x \mathrm{~d} y \leq \int_{\Omega}\left|\nabla^{2} u_{0}\right|^{2} \mathrm{~d} x \mathrm{~d} y .
$$


Trivially, $I_{\varepsilon}^{3}=0$. As for $I_{\varepsilon}^{2}$, we note that every coordinate square of side length $\varepsilon$ with center $z \in \Omega_{\varepsilon}$ with $t^{\varepsilon}-\varepsilon \sqrt{2} \leq \operatorname{dist}\left(z, K^{\prime \prime}\right) \leq t^{\varepsilon}+\varepsilon \sqrt{2}$ is contained in $D^{\varepsilon}$; hence, by (6.7) we can estimate

$$
\begin{aligned}
I_{\varepsilon}^{2} & \leq 4 \gamma \varepsilon \#\left(\left\{z \in \Omega_{\varepsilon}: t^{\varepsilon}-\varepsilon \sqrt{2} \leq \operatorname{dist}\left(z, K^{\prime \prime}\right) \leq t^{\varepsilon}+\varepsilon \sqrt{2}\right\}\right) \\
& \leq 4 \gamma \varepsilon \frac{1}{\varepsilon^{2}}\left|D^{\varepsilon}\right| \leq 24 \gamma \sqrt{2} \frac{\rho}{\rho-\delta-3 \varepsilon \sqrt{2}}\left(\mathcal{H}^{1}\left(K^{\prime \prime}\right)+o_{\rho}(1)\right) .
\end{aligned}
$$

Hence:

$$
\limsup _{\varepsilon \rightarrow 0} E_{\varepsilon}\left(u_{\varepsilon}^{\rho, \delta}, \Omega\right) \leq \int_{\Omega}\left|\nabla^{2} u_{0}\right|^{2} \mathrm{~d} x \mathrm{~d} y+24 \gamma \sqrt{2} \frac{\rho}{\rho-\delta}\left[\mathcal{H}^{1}\left(S_{u_{0}} \cup S_{\nabla u_{0}}\right)+o_{\rho}(1)\right] .
$$

Since $\lim _{\rho, \delta \rightarrow 0} \lim _{\varepsilon \rightarrow 0}\left\|u_{\varepsilon}^{\rho, \delta}-u_{0}\right\|_{L^{2}}=0$, letting $\rho$ and $\delta$ tend to 0 , by a diagonal argument we then have the thesis for $u_{0}$ satisfying (6.5) and (6.6).

The general case is obtained by approximation. In order to simplify the notation, we suppose without loss of generality that $\Omega$ is star-shaped with respect to the origin. Given an arbitrary target $u_{0}$, for fixed $\lambda>0$ we can define $v^{\lambda}$ as a minimizer of the energy

$$
v \mapsto \int_{\Omega}\left|\nabla^{2} v\right|^{2} \mathrm{~d} x \mathrm{~d} y+24 \gamma \sqrt{2} \mathcal{H}^{1}\left(S_{v} \cup S_{\nabla v}\right)+\lambda \int_{\Omega}\left|v-u_{0}\right|^{2} \mathrm{~d} x \mathrm{~d} y
$$

and $u^{\lambda}(x, y)=v^{\lambda}\left(\frac{\lambda}{1+\lambda}(x, y)\right)$. By the regularity results of $[20,21]$ such $u^{\lambda}$ satisfy conditions (6.5) and (6.6); moreover, after setting

$$
u_{0}^{\lambda}(x, y)=u_{0}\left(\frac{\lambda}{1+\lambda}(x, y)\right)
$$

by the minimality of $v^{\lambda}$ we have

$$
\begin{aligned}
& \left(1+\frac{1}{\lambda}\right)^{2} \int_{\Omega}\left|\nabla^{2} u^{\lambda}\right|^{2} \mathrm{~d} x \mathrm{~d} y+\left(1+\frac{1}{\lambda}\right)^{-1} 24 \gamma \sqrt{2} \mathcal{H}^{1}\left(S_{u^{\lambda}} \cup S_{\nabla u^{\lambda}}\right)+\lambda\left(1+\frac{1}{\lambda}\right)^{-2} \int_{\Omega}\left|u^{\lambda}-u_{0}^{\lambda}\right|^{2} \mathrm{~d} x \mathrm{~d} y \\
& \leq\left(1+\frac{1}{\lambda}\right)^{2} \int_{\left(1+\frac{1}{\lambda}\right) \Omega}\left|\nabla^{2} u^{\lambda}\right|^{2} \mathrm{~d} x \mathrm{~d} y+\left(1+\frac{1}{\lambda}\right)^{-1} 24 \gamma \sqrt{2} \mathcal{H}^{1}\left(\left(S_{u^{\lambda}} \cup S_{\nabla u^{\lambda}}\right) \cap\left(1+\frac{1}{\lambda}\right) \Omega\right) \\
& +\lambda\left(1+\frac{1}{\lambda}\right)^{-2} \int_{\left(1+\frac{1}{\lambda}\right) \Omega}\left|u^{\lambda}-u_{0}^{\lambda}\right|^{2} \mathrm{~d} x \mathrm{~d} y \\
& =\int_{\Omega}\left|\nabla^{2} v^{\lambda}\right|^{2} \mathrm{~d} x \mathrm{~d} y+24 \gamma \sqrt{2} \mathcal{H}^{1}\left(S_{v^{\lambda}} \cup S_{\nabla v^{\lambda}}\right)+\lambda \int_{\Omega}\left|v^{\lambda}-u_{0}\right|^{2} \mathrm{~d} x \mathrm{~d} y \\
& \leq\left(\int_{\Omega}\left|\nabla^{2} u_{0}\right|^{2} \mathrm{~d} x \mathrm{~d} y+24 \gamma \sqrt{2} \mathcal{H}^{1}\left(S_{u_{0}} \cup S_{\nabla u_{0}}\right)\right) .
\end{aligned}
$$

From this chain of inequalities we obtain first that $u^{\lambda}-u_{0}^{\lambda} \rightarrow 0$ in $L^{2}(\Omega)$, and hence that $u^{\lambda} \rightarrow u_{0}$ in $L^{2}(\Omega)$, as $\lambda \rightarrow+\infty$, and secondly that

$$
\int_{\Omega}\left|\nabla^{2} u^{\lambda}\right|^{2} \mathrm{~d} x \mathrm{~d} y+24 \gamma \sqrt{2} \mathcal{H}^{1}\left(S_{u^{\lambda}} \cup S_{\nabla u^{\lambda}}\right) \leq\left(1+\frac{1}{\lambda}\right)\left(\int_{\Omega}\left|\nabla^{2} u_{0}\right|^{2} \mathrm{~d} x \mathrm{~d} y+24 \gamma \sqrt{2} \mathcal{H}^{1}\left(S_{u_{0}} \cup S_{\nabla u_{0}}\right)\right) .
$$

From the first part of the proof, having set $E^{\prime \prime}(u)=\Gamma$ - $\limsup _{\varepsilon \rightarrow 0} E_{\varepsilon}(u)$ (see [13] Sect. 1.6), we have, taking into account (6.8)),

$$
E^{\prime \prime}\left(u_{\lambda}\right) \leq\left(1+\frac{1}{\lambda}\right)\left(\int_{\Omega}\left|\nabla^{2} u_{0}\right|^{2} \mathrm{~d} x \mathrm{~d} y+24 \gamma \sqrt{2} \mathcal{H}^{1}\left(S_{u_{0}} \cup S_{\nabla u_{0}}\right)\right)
$$


By the lower semicontinuity of the $\Gamma$-limsup (see [13] Sect. 1.7) we have

$$
E^{\prime \prime}\left(u_{0}\right) \leq \liminf _{\lambda \rightarrow+\infty} E^{\prime \prime}\left(u^{\lambda}\right) \leq \int_{\Omega}\left|\nabla^{2} u_{0}\right|^{2} \mathrm{~d} x \mathrm{~d} y+24 \gamma \sqrt{2} \mathcal{H}^{1}\left(S_{u_{0}} \cup S_{\nabla u_{0}}\right),
$$

as a consequence of (6.9).

Remark 6.3. A slightly more refined argument by slicing in the proof above for regular $u$ satisfying (6.5) and (6.6) gives an upper estimate with the anisotropic energy $\mathrm{BZ}_{\alpha, \beta}^{1}$ as defined in (3.3) with $\alpha=\beta=24 \sqrt{2} \gamma$. Unfortunately, the second part of the proof cannot be directly recovered from existence results in the literature.

The upper bound above is clearly not sharp, since it recovers both crease and jump discontinuities as limits of two layers of 'discrete' jump discontinuities. The need of this approximation comes from the difficulty of constructing optimal transitions close to general jump or crease sets. It can be overcome when all discontinuity sets are parallel to the coordinate axes. In this case we have the following result.

Theorem 6.4 (sharp bounds). Let $u$ be a piecewise $C^{2}$ function with $S_{u}$ and $S_{\nabla u}$ composed of segments parallel to the coordinate axes. Then there exists the $\Gamma$-limit

$$
\Gamma-\lim _{\varepsilon \rightarrow 0} E_{\varepsilon}(u)=\int_{\Omega}\left|\nabla^{2} u(x, y)\right|^{2} \mathrm{~d} x \mathrm{~d} y+2 \gamma \mathcal{H}^{1}\left(S_{u}\right)+\gamma \mathcal{H}^{1}\left(S_{\langle\nabla u, \nu\rangle} \backslash S_{u}\right)+2 \gamma \mathcal{H}^{1}\left(S_{\left\langle\nabla u, \nu^{\perp}\right\rangle}\right),
$$

where $S_{\langle\nabla u, \nu\rangle}$ is the subset of $S_{\nabla u}$ where the orthogonal component $\langle\nabla u, \nu\rangle$ of $\nabla u$ is discontinuous, and $S_{\left\langle\nabla u, \nu^{\perp}\right\rangle}$ is the subset of $S_{\nabla u}$ (possibly not disjoint from the previous one) where the tangential component $\left\langle\nabla u, \nu^{\perp}\right\rangle$ of $\nabla u$ is discontinuous.

Proof. The proof follows from a direct computation by choosing the (discretizations of) same target $u$ as recovery sequence, and noticing that it gives the lower bound obtained in Theorem 6.1.

\section{REFERENCES}

[1] R. Alicandro and M. Cicalese, A general integral representation result for continuum limits of discrete energies with superlinear growth. SIAM J. Math. Anal. 36 (2004) 1-37.

[2] R. Alicandro, M. Focardi and M.S. Gelli, Finite difference approximation of energies in fracture mechanics. Ann. Scuola Norm. Sup. Pisa Cl. Sci. 29 (2000) 671-709.

[3] L. Ambrosio and V.M. Tortorelli, Approximation of functionals depending on jumps by elliptic functionals via $\Gamma$-convergence. Comm. Pure Appl. Math. 43 (1990) 999-1036.

[4] L. Ambrosio and V.M. Tortorelli, On the approximation of free discontinuity problems. Boll. Un. Mat. Ital. B 6 (1992) $105-123$.

[5] L. Ambrosio, N. Fusco and D. Pallara, Functions of Bounded Variation and Free Discontinuity Problems. Oxford University Press, Oxford (2000).

[6] L. Ambrosio, L. Faina and R. March, Variational approximation of a second order free discontinuity problem in computer vision. SIAM J. Math. Anal. 32 (2001) 1171-1197.

[7] G. Bellettini and A. Coscia, Approximation of a functional depending on jumps and corners. Boll. Un. Mat. Ital. B 8 (1994) $151-181$.

[8] A. Blake and A. Zisserman, Visual Reconstruction. MIT Press, Cambridge, MA (1987).

[9] B. Bourdin and A. Chambolle, Implementation of an adaptive finite-element approximation of the Mumford-Shah functional. Numer. Math. 85 (2000) 609-646.

[10] M. Brady and B.K.P. Horn, Rotationally symmetric operators for surface interpolation. Computer Vision, Graphics, and Image Processing 22 (1983) 70-94.

[11] A. Braides, Lower semicontinuity conditions for functionals on jumps and creases. SIAM J. Math Anal. 26 (1995) $1184-1198$.

[12] A. Braides, Approximation of Free-discontinuity Problems. Springer Verlag, Berlin (1998).

[13] A. Braides, $\Gamma$-convergence for Beginners. Oxford University Press, Oxford (2002).

[14] A. Braides, Discrete approximation of functionals with jumps and creases, in Homogenization, 2001 (Naples) GAKUTO Internat. Ser. Math. Sci. Appl. 18. Tokyo, Gakkōtosho (2003) 147-153.

[15] A. Braides and M.S. Gelli, Limits of discrete systems with long-range interactions. J. Convex Anal. 9 (2002) $363-399$. 
[16] A. Braides and A. Piatnitski, Overall properties of a discrete membrane with randomly distributed defects. Arch. Ration. Mech. Anal. 189 (2008) 301-323.

[17] A. Braides, A.J. Lew and M. Ortiz, Effective cohesive behavior of layers of interatomic planes. Arch. Ration. Mech. Anal. 180 (2006) 151-182.

[18] A. Braides, M. Solci and E. Vitali, A derivation of linear elastic energies from pair-interaction atomistic systems. Netw. Heterog. Media 2 (2007) 551-567

[19] M. Carriero, A. Leaci and F. Tomarelli, A second order model in image segmentation: Blake and Zisserman functional, in Variational Methods for Discontinuous Structures (Como, 1994), Progr. Nonlin. Diff. Eq. Appl. 25, edited by R. Serapioni and F. Tomarelli. Basel, Birkhäuser (1996) 57-72.

[20] M. Carriero, A. Leaci and F. Tomarelli, Strong minimizers of Blake and Zisserman functional. Ann. Scuola Norm. Sup. Pisa Cl. Sci. 25 (1997) 257-285.

[21] M. Carriero, A. Leaci and F. Tomarelli, Density estimates and further properties of Blake and Zisserman functional, in From Convexity to Nonconvexity, Nonconvex Optim. Appl. 55, edited by R. Gilbert and Pardalos. Kluwer Acad. Publ., Dordrecht (2001) 381-392

[22] M. Carriero, A. Leaci and F. Tomarelli, Euler equations for Blake and Zisserman functional. Calc. Var. Partial Diff. Eq. 32 (2008) 81-110.

[23] M. Carriero, A. Leaci and F. Tomarelli, A Dirichlet problem with free gradient discontinuity. Adv. Mat. Sci. Appl. 20 (2010) $107-141$

[24] M. Carriero, A. Leaci and F. Tomarelli, A candidate local minimizer of Blake and Zisserman functional. J. Math Pures Appl. 96 (2011) $58-87$

[25] A. Chambolle, Un théorème de $\Gamma$-convergence pour la segmentation des signaux. C. R. Acad. Sci., Paris, Ser. I 314 (1992) 191-196.

[26] A. Chambolle, Image segmentation by variational methods: Mumford and Shah functional and the discrete approximations. SIAM J. Appl. Math. 55 (1995) 827-863.

[27] A. Chambolle, Finite-differences approximation of the Mumford-Shah functional. ESAIM: M2AN 33 (1999) $261-288$.

[28] A. Chambolle and G. Dal Maso, Discrete approximation of the Mumford-Shah functional in dimension two. ESAIM: M2AN 33 (1999) 651-672.

[29] P.G. Ciarlet, The Finite Element Method for Elliptic Problems. North-Holland Publishing Co., Amsterdam (1978).

[30] S. Conti, I. Fonseca and G. Leoni A $\Gamma$-convergence result for the two-gradient theory of phase transitions. Comm. Pure Appl. Math. 55 (2002) 857-936.

[31] S. Geman and D. Geman, Stochastic relaxation, Gibbs distributions, and the Bayesian restoration of images. IEEE PAMI 6 (1984) $721-724$.

[32] W.E.L. Grimson, From Images to Surfaces. The MIT Press Classic Series. MIT, Cambridge (1981).

[33] D. Mumford and J. Shah, Optimal approximations by piecewise smooth functions and associated variational problems. Comm. Pure Appl. Math. 42 (1989) 577-685.

[34] P. Santos and E. Zappale, Lower Semicontinuity in SBH. Mediterranean J. Math. 5 (2008) 221-235.

[35] B. Schmidt, On the derivation of linear elasticity from atomistic models. Netw. Heterogen. Media 4 (2009) 789-812. 